\title{
Radial distribution of the multiple stellar populations in $\omega$ Centauri ${ }^{\star}$
}

\author{
A. Bellini ${ }^{1,2}$, G. Piotto ${ }^{1}$, L. R. Bedin ${ }^{2}$, I. R. King ${ }^{3}$, J. Anderson ${ }^{2}$, A. P. Milone ${ }^{1}$, and Y. Momany ${ }^{4}$ \\ 1 Dipartimento di Astronomia, Università di Padova, Vicolo dell'Osservatorio 3, 35122 Padua, Italy \\ e-mail: [andrea.bellini; giampaolo.piotto; antonino.milone]@unipd.it \\ 2 Space Telescope Science Institute, 3700 San Martin Drive, Baltimore, MD 21218, USA \\ e-mail: [bellini; bedin; jayander]@stsci.edu \\ 3 Department of Astronomy, University of Washington, Seattle, WA 98195-1580, USA \\ e-mail: king@astro.washington.edu \\ 4 INAF - Osservatorio Astronomico di Padova, vicolo dell'Osservatorio 5, 35122 Padova, Italy \\ e-mail: yazan.almomany@oapd.inaf.it
}

Received 24 June 2009 / Accepted 23 September 2009

ABSTRACT

\begin{abstract}
Aims. We present a detailed study of the radial distribution of the multiple populations identified in the Galactic globular cluster $\omega$ Cen. Methods. We used both space-based images (ACS/WFC and WFPC2) and ground-based images (FORS1@VLT and WFI@ 2.2m ESO telescopes) to map the cluster from the inner core to the outskirts ( 20 arcmin). These data sets have been used to extract high-accuracy photometry for the construction of color-magnitude diagrams and astrometric positions of $\sim 900000$ stars.

Results. We find that in the inner $\sim 2$ core radii the blue main sequence (bMS) stars slightly dominate the red main sequence (rMS) in number. At greater distances from the cluster center, the relative numbers of bMS stars with respect to rMS drop steeply, out to $\sim 8$ arcmin, and then remain constant out to the limit of our observations. We also find that the dispersion of the Gaussian that best fits the color distribution within the bMS is significantly greater than the dispersion of the Gaussian that best fits the color distribution within the rMS. In addition, the relative number of intermediate-metallicity red-giant-branch stars (which includes the progeny of the bMS) with respect to the metal-poor component (the progeny of the rMS) follows a trend similar to that of the main-sequence star-count ratio $N_{\mathrm{bMS}} / N_{\mathrm{rMS}}$. The most metal-rich component of the red-giant branch follows the same distribution as the intermediatemetallicity component

Conclusions. We briefly discuss the possible implications of the observed radial distribution of the different stellar components in $\omega$ Cen.
\end{abstract}

Key words. globular clusters: general - globular clusters: individual: $\omega$ Cen [NGC 5139] - stars: evolution - stars: population II techniques: photometric

\section{Introduction}

The globular cluster (GC) $\omega$ Centauri is the most-studied stellar system of our Galaxy, but nevertheless one of the most puzzling. Its stars cover a wide range in metallicity (Cannon \& Stobie 1973; Norris \& Bessell 1975, 1977; Freeman \& Rodgers 1975; Bessell \& Norris 1976; Butler et al. 1978; Norris \& Da Costa 1995; Suntzeff \& Kraft 1996; Norris et al. 1996), with a primary component at $[\mathrm{Fe} / \mathrm{H}] \sim-1.7$ to -1.8 , and a long tail extending up to $[\mathrm{Fe} / \mathrm{H}] \sim-0.6$, containing three or four secondary peaks (see Johnson et al. 2009, for a recent update). It has been shown, both with ground-based photometry (Lee et al. 1999; Pancino et al. 2000; Rey et al. 2004; Sollima et al. 2005a; Villanova et al. 2007) and Hubble Space Telescope (HST) photometry (Anderson 1997; Bedin et al. 2004; Ferraro et al. 2004), that $\omega$ Cen hosts different stellar populations, most of them clearly visible in most of their evolutionary phases.

* Based on observations with the NASA/ESA Hubble Space Telescope, obtained at the Space Telescope Science Institute, which is operated by AURA, Inc., under NASA contract NAS 5-26555, and on observations made with ESO telescopes at La Silla and Paranal Observatories.
These populations have been linked to the aforementioned metallicity peaks in photometric studies of the red-giant branch (RGB) (Pancino et al. 2000; Hilker \& Richtler 2000; Sollima et al. 2005a), the subgiant branch (SGB) (Hilker et al. 2004; Sollima et al. 2005b; Stanford et al. 2006; Villanova et al. 2007), and the main sequence (MS) (Piotto et al. 2005). The most puzzling feature in $\omega$ Cen was discovered by Piotto et al. (2005), who showed that, contrary to any expectation from stellarstructure theory, the bluer of the two principal main sequences (bMS) is more metal-rich than the redder one (rMS). The only possible way of reconciling the spectroscopic observations with the photometric ones is to assume a high overabundance of $\mathrm{He}$ for the bluer MS (Bedin et al. 2004; Norris 2004; Piotto et al. 2005). How such a high He content could have been formed is still a subject of debate (see Renzini 2008 for a review).

One of the scenarios proposed to account for all the observed features of $\omega$ Cen is a tidal stripping of an object that was originally much more massive (Zinnecker et al. 1988; Freeman 1993; Dinescu et al. 1999; Ideta \& Makino 2004; Tsuchiya et al. 2004; Bekki \& Norris 2006; Villanova et al. 2007). In this scenario, the cluster was born as a dwarf elliptical galaxy, which was subsequently tidally disrupted by the Milky Way. Since all 
the populations of such a galaxy pass through the center, the nucleus would have been left with a mixture of all of them.

It has also been suggested (Searle 1977; Makino et al. 1991; Ferraro et al. 2002) that $\omega$ Cen could have been formed by mergers of smaller stellar systems. In apparent support of this scenario, Ferraro et al. (2002) claimed that the most metal-rich RGB component of $\omega$ Cen (RGB-a, following the nomenclature of Pancino et al. 2000) has a significantly different mean proper motion from that of the other RGB stars, and they concluded that RGB-a stars must have had an independent origin. However, Platais et al. (2003) showed that the proper-motion displacement seen could instead be an uncalibratable artifact of the plate solution. More recently Bellini et al. (2009), with a new CCD-based proper-motion analysis, were able to demonstrate that all $\omega$ Cen RGB stars share the same mean motion to within a few $\mathrm{km} \mathrm{s}^{-1}$. Anderson \& van der Marel (2009) also find that the lower-turnoff population (the analog of the RGB-a) shows the same bulk motion as the rest of the cluster. Thus there is no longer a reason to think this population to be kinematically distinct and an indication of a recent merger. Another indication that the cluster likely did not form by mergers can be found in the observation in Pancino et al. (2007) that all three RGB components share the cluster rotation, which would not be the case if different populations had different dynamical origins, or at least would require an unlikely degree of fine tuning.

While $\omega$ Cen was long thought to be the only cluster to exhibit a spread in abundances, we now know that it is not alone. M 54 also clearly exhibits multiple RGBs (Sarajedini \& Layden (1995); Siegel et al. 2007), SGBs (Piotto 2009), and has hints of multiple MSs. The complexity of M 54 makes good sense, because it coincides with the nucleus of the tidally disrupting Sagittarius dwarf-spheroidal galaxy. M 54 might be the actual nucleus or, more likely, it may represent a cluster that migrated to the nucleus as a result of dynamical friction (Bellazzini et al. 2008). $\omega$ Cen and M 54 are the two most massive GCs in our Galaxy, and it is quite possible that they are the result of similar - and peculiar - evolutionary paths (Piotto 2009). In any case, even $\omega$ Cen and M 54 are not the only clusters to exhibit non-singular populations. Exciting new discoveries, made in the last few years, clearly show that the GC multi-population zoo is quite populated, inhomogeneous, and complex.

Piotto et al. (2007) published a color-magnitude diagram (CMD) of the globular cluster NGC 2808, in which they identified a well-defined triple MS (D'Antona et al. 2005, had already suspected an anomalous broadening of the MS and had associated it with the three populations proposed by D'Antona \& Caloi 2004, to explain the complex horizontal branch (HB) of this cluster). Another globular cluster, NGC 1851, must have at least two distinct stellar populations. In this case, the observational evidence comes from the split of the SGB (Milone et al. 2008). There are other GCs which undoubtedly show a split in the SGB, like NGC 6388 (Moretti et al. 2009), M 22 (Piotto 2009; Marino et al. 2009), 47 Tuc (Anderson et al. 2009), which also shows a MS broadening, or in the RGB, like M4 (Marino et al. 2008). Recent investigations (Rich et al. 2004; Faria et al. 2007) suggest that also other galaxies might host GCs with more than one population of stars.

Multiple-population GCs offer observational evidence that challenges the traditional view. For half a century, a GC has been considered to be an assembly of stars that (quoting Renzini \& Fusi Pecci 1988): "represent the purest and simplest stellar populations we can find in nature, as opposed to field populations, which result from an admixture of ages and compositions". If we allow for the fact that all the GCs for which $\mathrm{Na}$ and
$\mathrm{O}$ abundances have been measured show a well defined $\mathrm{Na} / \mathrm{O}$ anti-correlation (Carretta et al. 2006, 2008), suggesting an extended star-formation process, and that 11 of the 16 intermediate-age Large Magellanic Cloud GCs have been found to host multiple populations (Milone et al. 2009), multipopulations in GCs could be more the rule than the exception. De facto, a new era in globular-cluster research has started, and understanding how a multiple stellar system like $\omega$ Cen was born and has evolved is no longer the curious study of an anomaly, but rather may be a key to understanding basic star-formation processes.

One way to understand how the multiple populations may have originated is to study the spatial distributions of the different populations, which might retain information about where they formed. In particular, theoreticians have been finding that if the second generation of stars is formed from an interstellar medium polluted and shocked by the winds of the first generation, then we would expect that the second generation would be more concentrated towards the center of the cluster than the first one (see D'Ercole et al. 2008; Decressin et al. 2008; Bekki \& Mackey 2009). In the first two of these references it is shown that in such a scenario the two generations of stars would interact dynamically and would homogenize their radial distributions over time. As such, spatial gradients represent a fading fossil record of the cluster's dynamical history.

Since $\omega$ Cen has such a long relaxation time (1.1 Gyr in the core and 10 Gyr at the half-mass radius, Harris 1996), it is one of the few clusters where we might hope to infer the star-formation history by studying the internal kinematics and spatial distributions of the constituent populations. These measurements will provide precious hints and constraints to allow theoreticians to develop more reliable GC dynamical models.

In a recent paper, Sollima et al. (2007) showed that the starcount ratio $N_{\mathrm{bMS}} / N_{\mathrm{rMS}}$ is flat beyond $\sim 12^{\prime}$, but that inward to $\sim 8^{\prime}$ it increases to twice the envelope value. Thus the bMS stars (i.e., the supposed "He-enriched" population) are more concentrated towards the center than the rMS, which is presumed to be the first generation. Unfortunately, Sollima et al. (2007) could not provide information about the trend of $N_{\mathrm{bMS}} / N_{\mathrm{rMS}}$ within $\sim 8^{\prime}$, which corresponds roughly to 2 half-mass radii $\left(r_{\mathrm{h}}\right)$.

On the other hand, the radial distribution of RGB subpopulations has been analyzed by many authors (Norris et al. 1997; Hilker \& Richtler 2000; Pancino et al. 2000, 2003; Rey et al. 2004; Sollima et al. 2005a; Castellani et al. 2007; Johnson et al. 2009). All these works agree that the intermediate-metallicity population (RGB-MInt) is more centrally concentrated than the more metal-poor one (RGB-MP). However, there is a disagreement about the most metal-rich population (RGB-a): Pancino et al. (2000), Norris et al. (1997), and Johnson et al. (2009) found that the most metal-rich stars (RGB-a) are as concentrated as the intermediate-metallicity ones, and consequently more concentrated than the most metal-poor stars, whereas Hilker \& Richtler (2000) and Castellani et al. (2007) considered the RGB-a component to be the least-concentrated population. (Since our work in progress was already favoring the former view over the latter, we were concerned to reach the definitive truth of this matter.)

In the present paper, we trace the radial distribution of the stars of $\omega$ Cen, both on the MS and in the RGB region. Our radial density analysis covers both the center and the outskirts of the cluster, taking advantage of the combination of four instruments on three different telescopes, and of our propermotion measurements on ground-based multi-epoch wide-field images (Bellini et al. 2009). In Sect. 2 we describe in detail the photometric data and the reduction procedures. Section 3 
presents our analysis of the radial distribution of the stars on the two MSs. In Sect. 4 we perform an analogous study for the RGB stars. A brief discussion follows in Sect. 5.

\section{Observations and data reductions}

To trace the radial distribution of the different stellar populations in $\omega$ Cen, we analyzed several data sets, from four different cameras. To probe the dense inner regions of the cluster we took advantage of the space-based high resolving power of HST, using both the Wide Field Channel (WFC) of the Advanced Camera for Surveys (ACS), and the Wide Field and Planetary Camera 2 (WFPC2). For the relatively sparse outskirts of the cluster, we instead made use of deep archival ground-based observations collected with the FORS1 camera of the ESO Very Large Telescope (VLT). In addition, to link all the different data sets into a common astrometric and photometric reference system, we used the Wide Field Imager (WFI) at the focus of the ESO $2.2 \mathrm{~m}$ telescope (hereafter WFI@2.2m). This shallower data set was also used to study the red-giant branch in the outskirts of the cluster.

Figure 1 shows the footprints of the data sets, centered on the recently determined accurate center of $\omega$ Cen: RA $=$ 13:26:47.24, Dec = -47:28:46.45 (J2000.0, Anderson \& van der Marel 2009). The red footprints are those of HST observations. The larger ones are the ACS/WFC data sets, a $3 \times 3$ mosaic centered on the cluster center and a single field $\sim 17^{\prime} \mathrm{SW}$ of the center. The smaller red field, $\sim 7^{\prime} \mathrm{S}$ of the center, was observed with WFPC2. Blue rectangles show the partially overlapping FORS1@VLT fields, extending from $\sim 6^{\prime}$ to $\sim 25^{\prime}$. The large field in magenta is the $\sim 33^{\prime} \times 33^{\prime}$ field-of-view of our WFI@2.2m proper-motion catalog (Bellini et al. 2009). The figure also shows the major and minor axes (solid lines), taken from van de Ven et al. (2006). We divided the field into four quadrants, centered on the major and minor axes. The quadrants are labeled with Roman numerals and separated by dashed lines. We will use them to derive internal estimates of the errors of the star-count distribution. Concentric ellipses, aligned with the major/minor axes, have ellipticity of 0.17 , coincident with the average ellipticity of $\omega$ Cen (Geyer et al. 1983). These ellipses will be used to define radial annuli, in Sect. 2.8. Thick black circles mark the core radius $\left(r_{\mathrm{c}}=11^{\prime} 4\right)$ and the half-mass radius $\left(r_{\mathrm{h}}=4{ }^{\prime} .18\right)$ (from Harris 1996). If we assuming a cluster distance of $4.7 \mathrm{kpc}$ (van de Ven et al. 2006; van der Marel \& Anderson 2009), the two radii correspond to $1.9 \mathrm{pc}$ and $5.7 \mathrm{pc}$, respectively.

The details of the data sets are summarized in Table 1. In the following subsections we give brief descriptions of the reduction procedures, which have been presented in more detail in various other papers. The FORS1 data, however, were taken by Sollima et al. (2007), for a purpose similar to ours; we will give a full description of our reduction in Sect. 2.4.

\subsection{HST: ACS/WFC inner $3 \times 3$ mosaic}

This data set (inner nine red rectangles in Fig. 1, GO-9442, PI Cool) consists of a mosaic of $3 \times 3$ fields obtained with the ACS/WFC through the F435W and F625W filters. This camera has a pixel size of $\sim 50$ mas and a field of view of $3 ! 3 \times 3 ! 3$. Each of these nine fields has one short and three long exposures in both F435W and F625W. The mosaic covers the inner $\sim 10^{\prime} \times 10^{\prime}$, the most crowded region of $\omega$ Cen. These images, which were used by Ferraro et al. (2004) and by Freyhammer et al. (2005), and which we used in both Bedin et al. (2004), and Villanova et al. (2007), were reduced using img2xym_WFC.09x10, which is a publicly available

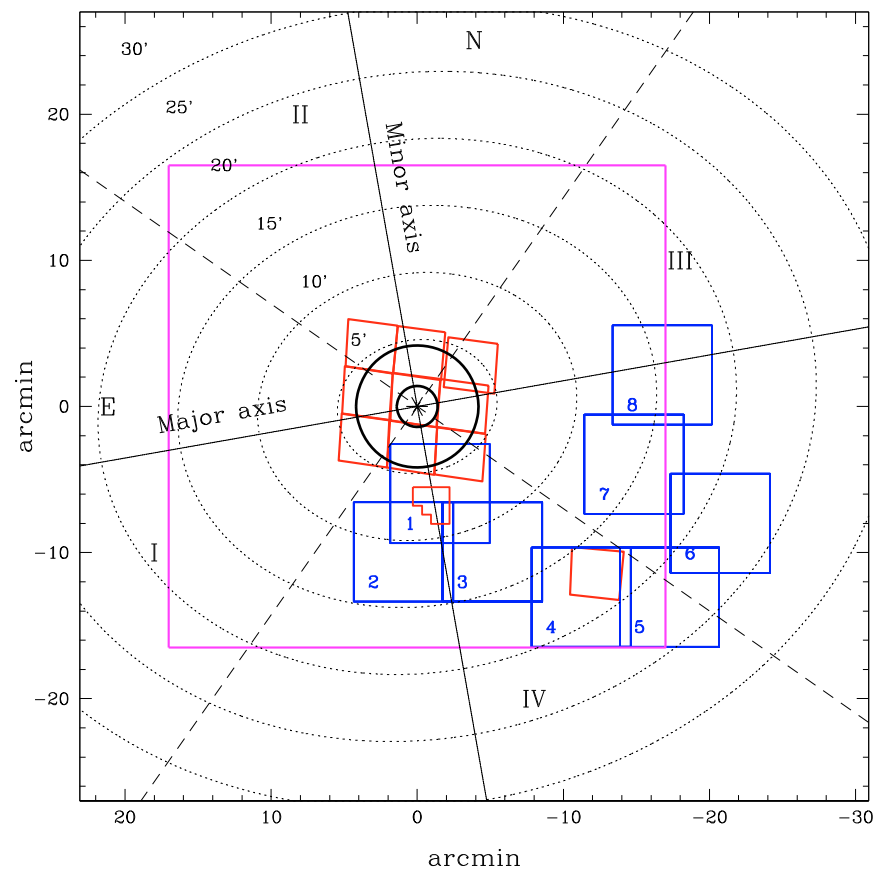

Fig. 1. The footprints of the $\omega$ Cen data sets used in this work. North is up, east to the left. The " $*$ " marks the cluster center. The $3 \times$ 3 ACS/WFC mosaic (in red) is that of GO-9442, while the 8 FORS1 fields are marked in blue. The largest field (in magenta) comes from the WFI proper-motion catalog (Bellini et al. 2009). This wide-field catalog has been used to register the FORS1 and the ACS/WFC innermosaic data into a common astrometric and photometric reference system (see text). The smaller WFPC2 field at $\sim 7^{\prime}$ south and the outer ACS/WFC field at $\sim 17^{\prime}$ from the cluster center are also shown (in red). Concentric ellipses, centered on the center of $\omega$ Cen and aligned with the major and minor axes, show the radial bins that we created. Ellipses are split into quadrants (dashed lines), labeled with Roman numerals. Each quadrant is bisected by the major or minor axis. Thick black circles mark the core radius $\left(r_{\mathrm{c}}=1\right.$ !. 4$)$ and the half-mass radius $\left(r_{\mathrm{h}}=4\right.$ !.18) (from Harris 1996).

Table 1. Data sets used in this work.

\begin{tabular}{clr}
\hline \hline Data set & Filter & \# images $\times$ Exp. time $(\mathrm{s})$ \\
\hline $\begin{array}{c}3 \times 3 \text { ACS/WFC } \\
\text { inner mosaic }\end{array}$ & F435W & $27 \times 340,9 \times 12$ \\
& F625W & $27 \times 340,9 \times 8$ \\
ACS/WFC & F606W & $2 \times 1285,2 \times 1300$, \\
$\sim 17^{\prime}$ & & $2 \times 1331,2 \times 1375$ \\
& F814W & $4 \times 1331,2 \times 1340,2 \times 1375$ \\
WFPC2@HST & F606W & $2 \times 300,1 \times 600$ \\
$\sim 7^{\prime}$ & F814W & $2 \times 400,1 \times 1000$ \\
FORS1@VLT & $B$ & $20 \times 1100$ \\
WFI@2.2m & $B$ & $1 \times 10,1 \times 15,11 \times 30,1 \times 40$, \\
& $B$ & $1 \times 60,1 \times 120,2 \times 240,2 \times 300$ \\
& $R_{\mathrm{C}}$ & $1 \times 5,1 \times 10,1 \times 15,1 \times 30,5 \times 60$ \\
& $V$ & $6 \times 5,9 \times 10,1 \times 15,3 \times 20$, \\
& & $10 \times 90,7 \times 120,1 \times 150,3 \times 240$ \\
\hline
\end{tabular}

FORTRAN program, described in Anderson \& King (2006). The program finds and measures each star in each exposure by fitting a spatially-variable effective point-spread function. The independent measurements of the stars were collated into a master star list that covers the entire $3 \times 3$ mosaic field. For each star we constructed an average magnitude in each band, and computed 
the rms deviation of the multiple measurements about this average. Instrumental magnitudes were transformed into the ACS Vega-mag flight system following the procedure given in Bedin et al. (2005), using the zero points of Sirianni et al. (2005). Since the zero points are valid only for fluxes in the _drz exposures, we computed calibrated photometry for a few isolated stars in the _drz exposures and used this to set the zero points for the photometry that was based on the individual _flt images. Saturated stars in short exposures were treated as described in Sect. 8.1 in Anderson et al. (2008). Collecting photoelectrons along the bleeding columns allowed us to measure magnitudes of saturated stars up to $3.5 \mathrm{mag}$ above saturation (i.e., up to $m_{\mathrm{F} 435 \mathrm{~W}} \simeq 12 \mathrm{mag}$ ), with errors of only a few percent (Gilliland 2004). We used the final catalog, which contains more than 760000 stars, to trace the radial distribution of RGB and MS stars in this most crowded region of the cluster.

\subsection{HST: ACS/WFC outer field}

The outer ACS field ( $\sim 17^{\prime} \mathrm{SW}$ of the cluster center, see Fig. 1) comes from proposals GO-9444 and GO-10101 (both with PI King), using the F606W and F814W filters. The photometry from the first-epoch observations was published in Bedin et al. (2004). The photometry presented in the present paper comes from the full two-epoch data set for this field; the two epochs also allow us to derive proper motions and perform a critical cluster/field separation. A detailed description of the data reduction, the proper-motion measurement, and the resulting CMDs will be presented in a forthcoming paper. The reduction and calibration of these data sets use procedures similar to those used for the central mosaic, and provided photometry for $\sim 3500$ stars.

\subsection{HST: WFPC2 field}

We also make use of one WFPC2 field, $\sim 7^{\prime}$ south of the cluster center (see Fig. 1). This data set consists of $2 \times 300+$ $600 \mathrm{~s}$ exposures in F606W, and $2 \times 400+1000 \mathrm{~s}$ in F814W (GO-5370, PI Griffiths), and contains 9214 stars. These images have been reduced with the algorithms described in Anderson \& King (2000). The field was calibrated to the photometric Vegamag flight system of WFPC2 according to the prescriptions of Holtzman et al. (1995). This WFPC2 field is particularly important in tracing the distribution of stars in the MS of $\omega$ Cen, because it is at a radial distance from the center of the cluster where there are no suitable ACS/WFC observations and where groundbased observations are almost useless because of crowding.

\subsection{VLT: eight FORS1 fields}

The VLT data set consists of eight partially overlapping FORS1 fields, each with a pixel size of 200 mas and a field of view of $6{ }^{\prime} 8 \times 6{ }^{\prime} .8$. These fields (the blue rectangles in Fig. 1) probe the regions between $6^{\prime}$ and $25^{\prime}$ from the center of $\omega$ Cen. The set of images consists of $20 \times 1100 \mathrm{~s}$ exposures in $B$, and $20 \times 395 \mathrm{~s}$ in $R$, and are the same images used by Sollima et al. (2007). There are four images in each field (two per filter), except that the third and fourth fields have four images per filter (see Fig. 1 for field numbers). This is the only data set that we reduced specifically for the present work. For this reason we give a more detailed description of our reduction procedure.

We retrieved the data sets from the ESO archive; masterbias and flat-field frames were constructed using standard IRAF routines. Photometric reduction of the images was performed
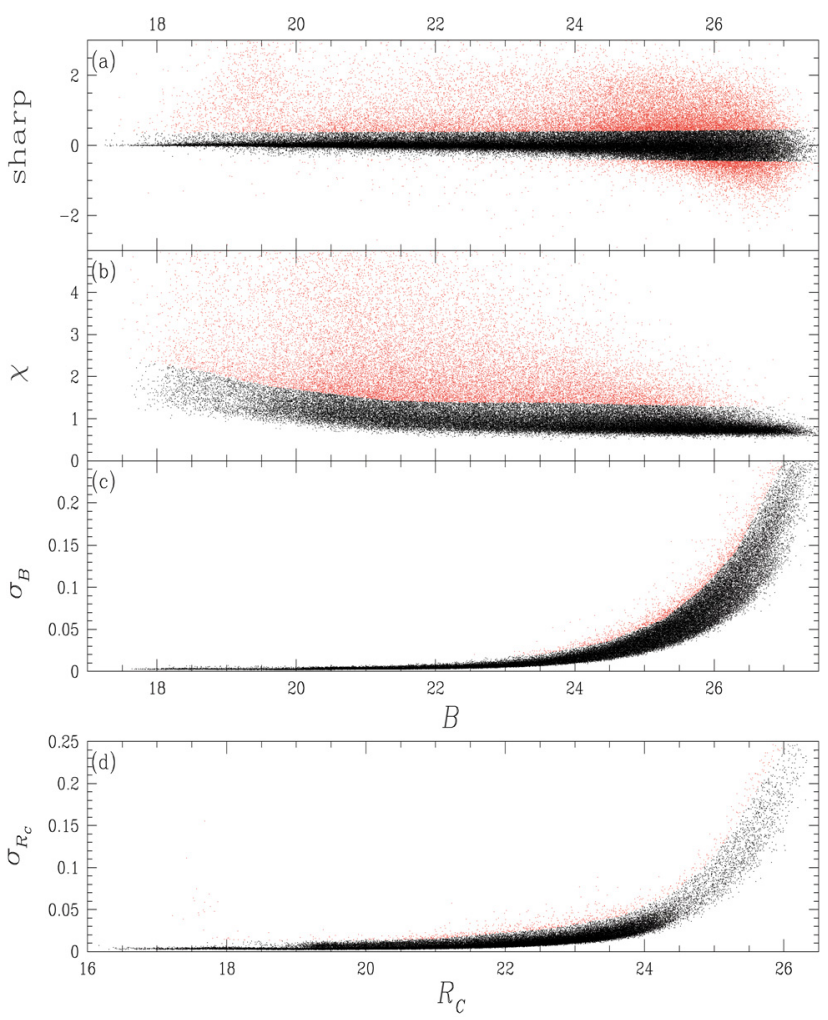

Fig. 2. Selection criteria used to isolate FORS1@VLT stars for our MS subpopulation analysis. Panel (a) shows sharp values versus $B$ magnitude, and panel (b) $\chi$ versus $B$. Panels (c) and (d) show the photometric errors as a function of the $B$ and Cousins- $R_{\mathrm{C}}$ magnitudes respectively. Only stars that passed the sharp selection criterion (black in the first panel), are plotted in the subsequent panels; similarly, only stars that also survived the $\chi$ selection are shown in the remaining two panels.

using Stetson's DAOPHOT-ALLSTAR-ALLFRAME packages (Stetson 1987, 1994). For each exposure we constructed a quadratic spatially variable point-spread function (PSF) by using a Penny function ${ }^{1}$, and for each individual exposure we chose by visual inspection - the best 100 (at least) isolated, bright, unsaturated stars that were suitable for mapping the PSF variations all over the image. We used ALLFRAME on each individual field, keeping only stars measured in at least four images. The photometric zero points of each field were registered to the instrumental magnitudes of the fourth field (the less crowded of the two that have more exposures). Finally, photometric and astrometric calibration was performed using the WFI@ $2.2 \mathrm{~m}$ astrometric-photometric catalog by Bellini et al. (2009) as a reference. As a result, we brought the FORS1 $R$ magnitudes to the Cousins- $R_{\mathrm{C}}$ photometric system used by WFI@2.2m. Our final FORS 1 catalog contains $\sim 133000$ objects.

Since the innermost FORS1 field is seriously affected by crowding, we did not use it in the present analysis. Figure 2 plots the sharp, $\chi$, and $\sigma_{B}$ and $\sigma_{R_{\mathrm{C}}}$ calculated by ALLFRAME, as functions of stellar magnitude, for the stars in the FORS1 catalog. To choose the well-measured stars, we drew by eye the cut-off boundaries in the quality parameters that retained objects

\footnotetext{
1 A Penny function is the sum of a Gaussian and a Lorentz function. In this case we used five free parameters: half-width at half-maximum of the Penny function, in the $x$ and in the $y$ coordinate; the fractional amplitude of the Gaussian function at the peak of the stellar profile; the position angle of the tilted elliptical Gaussian; and a tilt of the Lorentz function in a different direction from the Gaussian. The Lorentz function may be elongated too, but its long axis is parallel to the $x$ or $y$ direction.
} 
that were most likely to be well-measured stars. Panel (a) shows sharp values versus $B$ magnitude. Stars that passed the selection criterion are shown in black. Panel (b), which includes only stars that passed the sharp cut, shows $\chi$ values versus $B$. Stars that also passed the $\chi$ criterion are in black. In panel (c) we plot the $\sigma_{B}$ values versus $B$, for the stars that survived these two selections. Again, the stars with good photometry are shown in black. Finally, in the last panel we plot $\sigma_{R_{\mathrm{C}}}$ values versus $R_{\mathrm{C}}$, for all the survivors, and we highlight in black those that survived this selection too. At the end of these selection procedures, we are left with a catalog of $\sim 66500$ stars. We note that while these selection criteria affect stars at different magnitudes differently, they should not affect the ratio of stars on the bMS and rMS, since at a given magnitude the two populations should both have about the same photometric error, and the same probability of making it into our catalog.

\subsection{WFI@2.2m}

This data set was collected at the $2.2 \mathrm{~m}$ ESO Telescope, with the WFI camera, between 1999 and 2003. The WFI@2.2m camera is made up of a mosaic of $4 \times 2$ chips, $2048 \times 4096$ pixels each, with a pixel scale of 238 mas/pixel). Thus, each WFI exposure covers $\sim 34^{\prime} \times 33^{\prime}$. The $\omega$ Cen astrometric, photometric, and proper-motion catalog based on this data set and presented in Bellini et al. (2009) is public, and contains several wide-band $\left(U, B, V, R_{\mathrm{C}}, I_{\mathrm{C}}\right)$ filters plus a narrow-band filter $(658 \mathrm{~nm})$, and covers an area of $\sim 33^{\prime} \times 33^{\prime}$ centered on the cluster center. We refer the reader to Bellini et al. (2009) for a detailed discussion of the data-reduction and calibration procedures.

Briefly, photometry and astrometry were extracted with the procedures and codes described in Anderson et al. (2006). Photometric measurements were corrected for "sky concentration" effects ${ }^{2}$ and for differential reddening, as described in Manfroid \& Selman (2001) and Bellini et al. (2009). Global star positions are measured to better than $\sim 45$ mas in each coordinate. Photometric calibration in the $B, V, R_{\mathrm{C}}, I_{\mathrm{C}}$ bands is based on a set of $\sim 3000$ secondary standard stars in $\omega$ Cen, available on-line (Stetson 2000, 2005). Color equations were derived to transform our instrumental photometry into the photometrically calibrated system using an iterative least-squares linear fit. Thanks to the four-year time-baseline, we were able to successfully separate cluster members from field stars by means of the local-transformation approach (Anderson et al. 2006), giving us

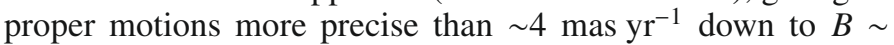
$20 \mathrm{mag}$, for $\sim 54000$ stars.

\subsection{The astrometric and photometric reference frame}

The large field of view of the WFI@2.2m camera makes our WFI catalog an ideal photometric and astrometric reference frame to which to refer all the other observations, from different telescope-camera-filter combinations. For each catalog we made the tie-in by means of stars that were in common with the reference catalog. For positions we derived a general six-parameter linear transformation to the astrometric system of the WFI catalog. For photometry we used as a reference standard the $B$ and Cousins- $R_{\mathrm{C}}$ magnitudes of the WFI@2.2m catalog, and transformed the magnitudes of each other catalog to this standard. For the $m_{\mathrm{F} 435 \mathrm{~W}}$ and $m_{\mathrm{F} 625 \mathrm{~W}}$ magnitudes of the central mosaic of $3 \times 3$ ACS/WFC fields, we used $\sim 3300$ stars that had been

\footnotetext{
${ }^{2}$ Light contamination caused by internal reflections of light in the optics, causing a redistribution of light in the focal plane.
}
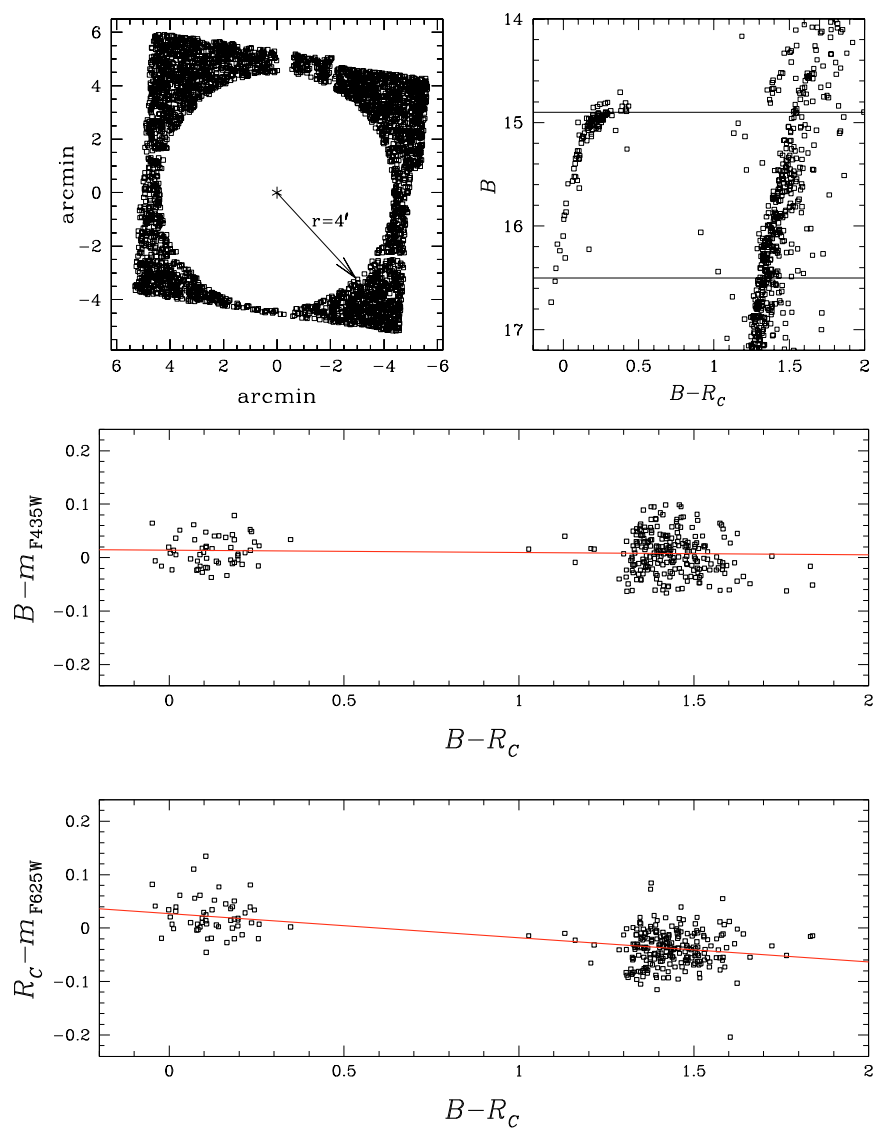

Fig. 3. (Top left:) selected stars in common between the ACS/WFC $3 \times 3$ mosaic and the WFI@2.2m data sets. (Top right:) horizontal lines mark the magnitude interval used to derive calibration equations. (Bottom panels:) calibration fits used to transform Vega-mag ACS/WFC $m_{\mathrm{F} 435 \mathrm{~W}}$ and $m_{\mathrm{F} 625 \mathrm{~W}}$ magnitudes into the WFI@2.2m photometric system. See text for details.

observed in common, located outside $4^{\prime}$ from the cluster center to avoid the most crowded regions in the WFI data set (top-left panel of Fig. 3). We excluded from this sample saturated stars in the WFI data set, keeping only the brighter $(14.9<B<16.5)$ and well measured $\left(\sigma_{B, R_{C}}<0.02 \mathrm{mag}\right)$ ones (top-right panel in Fig. 3). The adopted calibration fits are shown in the bottom panels of Fig. 3. We did similarly for the FORS1 $B$ and $R$ magnitudes.

Calamida et al. (2005) measured a differential reddening of up to $E(B-V) \sim 0.14$ in a region of $\sim 14^{\prime} \times 14^{\prime}$ centered on $\omega$ Cen. This result has been questioned by Villanova et al. (2007); in their Figs. 1-6, the sharpness of the SGB sequences suggests that the existence of any serious differential reddening is very unlikely. But in any case, a proper radial-distribution analysis needs correction even for a differential reddening that is of the order of few hundredths of a magnitude. Our corrections for differential reddening followed the method outlined by Sarajedini et al. (2007), which uses the displacements of individual stars from a fiducial sequence to derive a reddening map.

The outer ACS/WFC field at $\sim 17^{\prime}$ from the cluster center and the WFPC2 field at $\sim 7^{\prime}$ provide stellar photometry in the F606W and F814W bands. For the ACS field we have overlap with the WFI catalog, which allows us to calibrate the photometry, but the stars available are all on the main sequence above $m_{\mathrm{F} 606 \mathrm{~W}}=$ 21 , so they have a very narrow range in color, and we cannot empirically determine the color term in the calibration. For the WFPC2 field, in addition to the problem of the limited color 


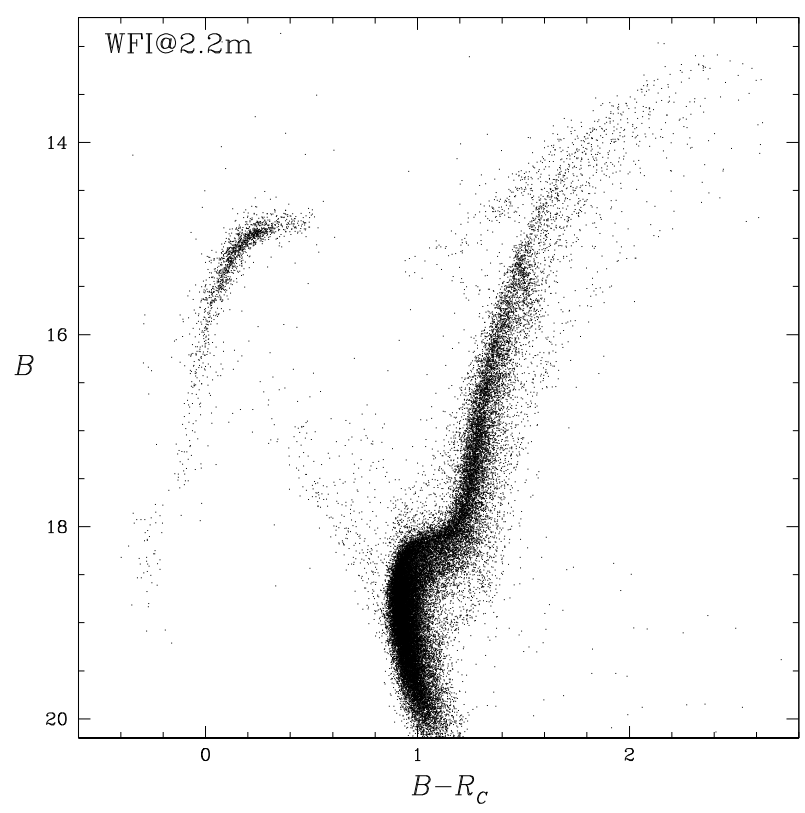

Fig. 4. $B$ vs. $B-R_{\mathrm{C}}$ CMD of WFI@2.2m stars, after calibration and proper-motion selection (see Bellini et al. 2009).

baseline, the WFI photometry in this inner field is of low quality on account of ground-based crowding. For these reasons, we decided to not transform the photometry of these two fields into the photometric reference system of WFI@2.2m, but dealt with them in the HST Vega-mag flight system.

\subsection{The deep color-magnitude diagrams}

Our proper-motion-selected WFI@2.2m $B$ vs. $B-R_{\mathrm{C}} \mathrm{CMD}$ is shown in Fig. 4. All the main features of the cluster are clearly visible, except for the split MS, since the WFI data go down only a magnitude or so below the turnoff. The CMDs of the other data sets that we analyzed are presented in Fig. 5, where the top-left panel refers to the eight FORS1@VLT fields, the middle-left panel to the proper-motion-selected CMD of the external ACS/WFC, the bottom-left panel to the CMD from the WFPC2 field, and the right panel of Fig. 5 to the CMD of the inner $3 \times 3 \mathrm{ACS} / \mathrm{WFC}$ mosaic. It is clear that the MS population can be studied in all but the WFI CMD, and the RGB population can be studied in the WFI and inner ACS data sets.

Now that we have a comprehensive sample of $\omega$ Cen stars, both for the bright stars and for the faint ones, covering the central region all the way out to $\sim 25^{\prime}$, we can define robust selection criteria for the subpopulations to track how the population fractions vary with radius.

\subsection{The angular radial distance: $r^{*}$}

Since $\omega$ Cen is elongated in the plane of the sky, it does not make sense to analyze its radial profile via circular annuli. We therefore decided to extract radial bins in the following way. We adopted the position angle (PA) of $100^{\circ}$ for the major-axis (van de Ven et al. 2006), and an average ellipticity of 0.17 (Geyer et al. 1983). To define the bins of the radial distribution we adopted elliptical annuli, whose major axes are aligned with the $\omega$ Cenmajor axis, and stars were extracted accordingly (see
Fig. 1). To indicate the angular radial distance from the cluster center, we used the equivalent radius $r^{*}$, defined as the radius of the circle with the same area as the corresponding ellipse (i.e., the geometrical mean of the semi-major and semi-minor axes). Each of the small fields (the outer ACS field and the WFPC2 field), we considered as a single radial bin.

\section{MS subpopulations}

Our goal in putting together these varied catalogs is to quantify the differences in the radial profiles of the various subpopulations of $\omega$ Cen. One way to do this would be to measure the surface density profile for each group and compare them directly, but this would require accurate completeness corrections and careful attention to magnitude bins. Since our interest, however, is simply to determine how the populations vary with respect to each other, we need only to measure the ratio of the populations as a function of radius. This ratio should be independent of completeness corrections and the details of the magnitude bins used, since the bMS and rMS differ only slightly in color and are observed over the same magnitude range.

Our analysis of the $N_{\mathrm{bMS}} / N_{\mathrm{rMS}}$ ratio is based mostly on the data sets from the inner ACS/WFC $3 \times 3$ mosaic and FORS1@VLT, which allow us to map the ratio of bMS/rMS from the cluster center out to $\sim 25^{\prime}$, once the photometry and astrometry have been brought into the same reference system. The other two fields, each of which covers only a small region, provide only one point each in our analysis of $N_{\mathrm{bMS}} / N_{\mathrm{rMS}}$ versus radius. Moreover, since we were not able to bring $m_{\mathrm{F} 606 \mathrm{~W}}$ and $m_{\mathrm{F} 814 \mathrm{~W}}$ photometry of the outer ACS and the WFPC2 field into the WFI $B$ and $R_{\mathrm{C}}$ photometric system, we kept the WFPC2 and the outer ACS/WFC data sets in their native photometric system, and used them only for a further (though important) confirmation of the radial gradient found with the FORS1 and inner ACS/WFC data sets.

\subsection{Straightened main sequences}

In order to analyze the color distribution of the stars along the MS in a more convenient coordinate system, we adopted a technique previously used with success in $\omega$ Cen (Anderson 1997 , 2002), and in other works (Sollima et al. 2007; Villanova et al. 2007; Piotto et al. 2007; Milone et al. 2008; Anderson et al. 2009).

We defined fiducial lines in the CMDs (drawn by hand), such as to be equidistant from the ridge lines of the bMS and rMS stars. We avoided choosing the ridge line of either sequence as our fiducial line, because we wanted a system in which both the sequences are as parallel and as rectified as possible. We used different fiducial lines for the $B, B-R_{\mathrm{C}}$ CMDs of the inner ACS/WFC and the FORS1 data sets and for the $m_{\mathrm{F} 606 \mathrm{~W}}$, $m_{\mathrm{F} 606 \mathrm{~W}}-m_{\mathrm{F} 814 \mathrm{~W}} \mathrm{CMDs}$ of the WFPC 2 and outer ACS/WFC data sets. In this way, we were sure to straighten the MSs in the same consistent way for the two different sets of filters. Then we subtracted from the color of each star the color of the fiducial line at the same luminosity as the star.

In Fig. 6 we show the CMDs in the $\omega$ Cen MS region for the central mosaic of ACS/WFC data (left panel), the FORS1@VLT (middle panel), and the WFPC2 $\sim 7^{\prime}$ field and the ACS/WFC field at $\sim 17^{\prime}$ (right panels). In the case of the central ACS/WFC data, we plotted only a randomly chosen $8 \%$ of the stars, in order to show the two sequences clearly. In all the CMDs the MS splitting is clearly visible. For the inner 

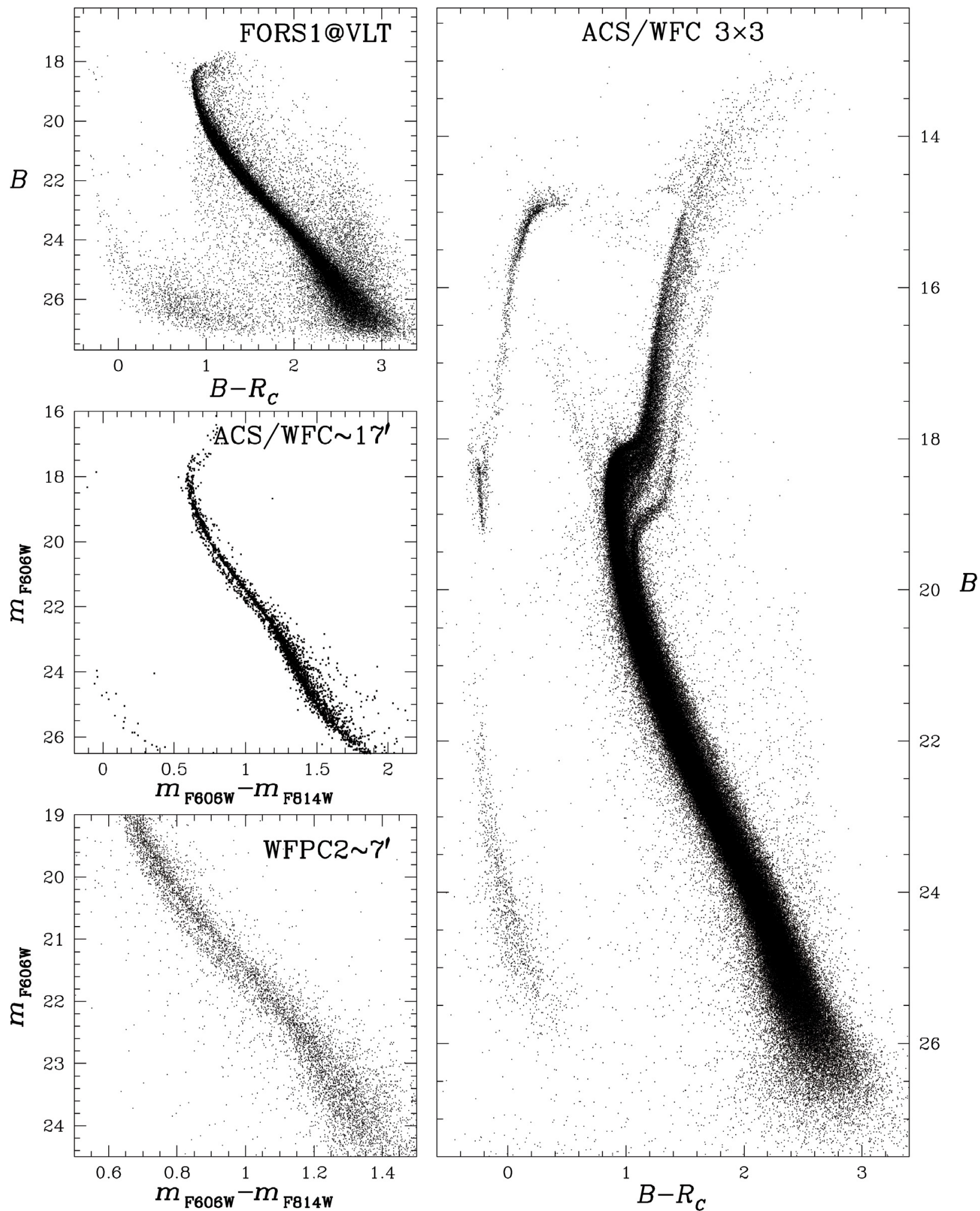

Fig. 5. (Top left): CMD from the eight FORS1@VLT fields. We can measure stars from the bottom of the RGB down to $B \sim 27.5$ mag. (Middle left): proper-motion-selected CMD from the outer ACS/WFC field. (Bottom left): CMD from the WFPC2 images located $\sim 7^{\prime}$ south of the cluster center. (Right panel): CMD of the inner $3 \times 3$ ACS/WFC fields. In the top left and the right-hand CMDs, the bMS and rMS fail to show separately only because the profusion of points blackens their whole region.

ACS/WFC and FORS1 data sets we restricted our MS analysis to the magnitude range $20.9 \leq B \leq 22.1$ (dashed lines in Fig. 6), the interval in which the two MSs are most separated in color and are parallel. For the same reasons we analyzed stars in the magnitude range $20.6 \leq m_{\mathrm{F} 606 \mathrm{~W}} \leq 21.9$ for the WFPC2 and the outer ACS/WFC data sets. The bright limit also avoids the saturated stars in the deep WFC exposures. The adopted fiducial lines are again plotted (in red in the color version of the paper). 


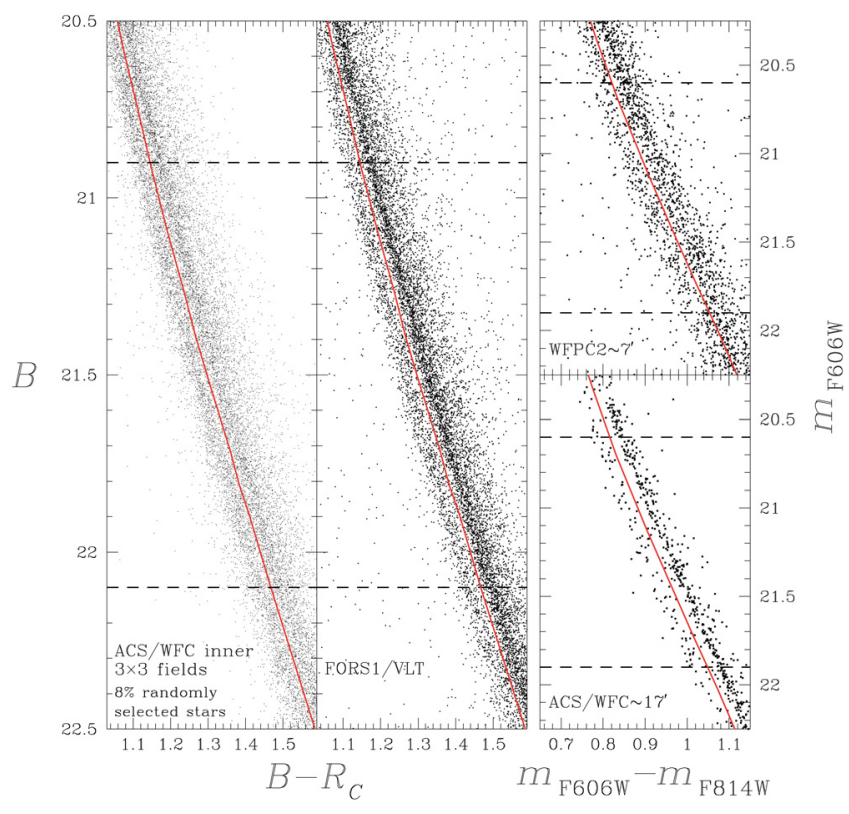

Fig. 6. The left panel shows a randomly selected $8 \%$ of the stars in the CMD of the inner $\sim 10^{\prime} \times 10^{\prime}$ ACS/WFC images, in the region of the MS where the two branches are most separated in color. The middle panel shows the CMD of the FORS1@VLT fields. The right panels show the outer ACS/WFC field (bottom) and WFPC2 field (top). The MS duality is clearly detected in all diagrams (see also Fig. 7). The dashed horizontal lines mark the selected magnitude range for the definition of the bMS and rMS samples used in the derivation of their radial profiles. The fiducial lines (drawn by hand) that were used to straighten and separate the sequences are also plotted (in red in the color version).

In Fig. 7 we show straightened CMDs for the same data sets shown in Fig. 6, with the only difference being that we now plot a $20 \%$ randomly generated sample of stars for the inner ACS/WFC data set, since the expanded color baseline allows more points to be seen. It is worth noting that even a simple inspection shows the $N_{\mathrm{bMS}} / N_{\mathrm{rMS}}$ ratio clearly decreasing as we go from the central cluster regions to the outer ones. It is also clear that the spread in the bMS is somewhat greater than that of the rMS.

Finally, note that we call the color deviation of a star from the fiducial line $\Delta\left(B-R_{\mathrm{C}}\right)$. We shall use this notation frequently in what follows.

Our aim in selecting the best-measured stars in the previous sections was so that we would be able to assign the stars to the different populations as accurately as possible. Similarly, as much as possible we transformed our photometry into the same system, so that our population selections throughout the cluster would be as consistent as possible.

Even with these careful steps, however, it is still difficult to ensure that we are selecting stars of the same population in the inner parts of the cluster as in the outer parts. Even if we had observations with the same detector at all radii, the greater crowding at the center would increase the errors there. On the other hand, our use of ground-based images for the outer fields actually makes those fields even more vulnerable to crowding effects.

Another complication comes from main-sequence binaries, which at the distance of a globular cluster are unresolved. Relaxation, causing mass segregation, will concentrate them to the cluster center and cause a redward distortion of the main sequence there.

Moreover, in the lower-density outer regions of the cluster we can get the same statistical significance only by using larger

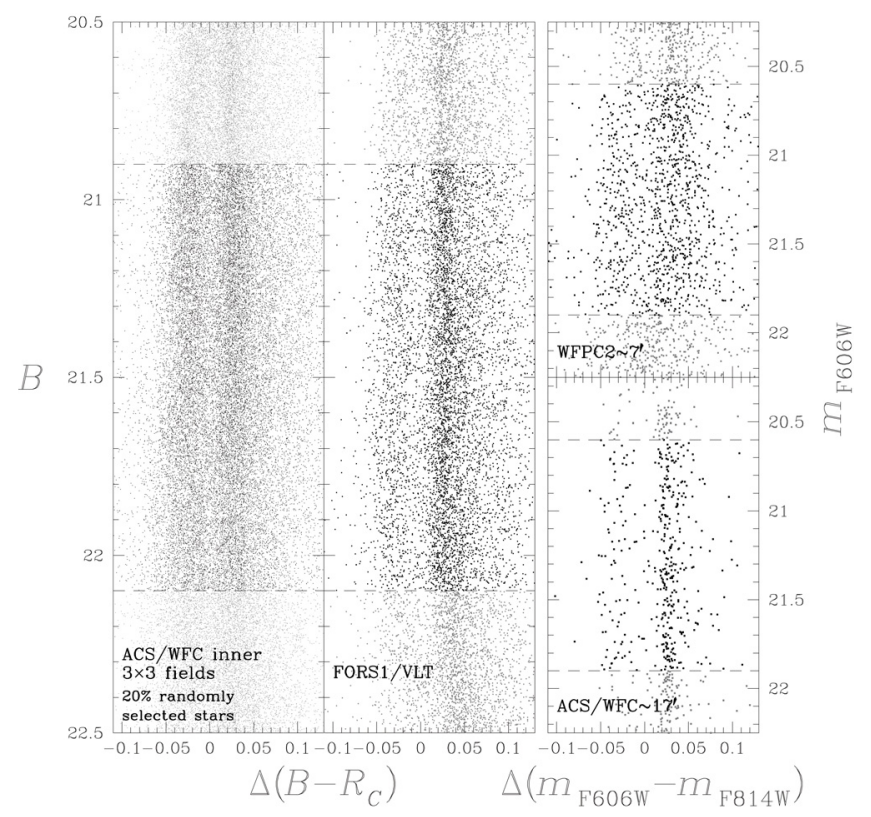

Fig. 7. Same as Fig. 6, but after subtraction, from the color of each star, of the color of the fiducial line at the same luminosity. In the left panel we show a randomly selected $20 \%$ of the stars from the ACS/WFC central-mosaic data (rather than the previous $8 \%$, since the color-scale is now less compressed).

areas, with an increased vulnerability to inclusion of field stars. Finally, the red side of the main sequence is contaminated by the anomalous metal-rich population (hereafter MS-a), which is clearly connected with RGB-a. Even if these stars include only $\sim 5 \%$ of the total cluster members (Lee et al. 1999; Pancino et al. 2000; Sollima et al. 2005a; Villanova et al. 2007), they are an additional source of pollution for rMS stars - against which we now take specific precautions.

\subsection{Dual-Gaussian fitting}

There is no way of dealing with the above issues perfectly, but we did our best to make our measurements as insensitive to them as possible. To this end, we measured the bMS and rMS fractions by simultaneously fitting the straightened color distributions with two Gaussians, and taking the area under each Gaussian as our estimate of the number of stars in each population. By keeping the width of each Gaussian an adjustable parameter, we allowed in a natural way for the fact that the photometric scatter differs from one radius and data set to another.

While the dual Gaussians provide a natural way of measuring the two populations in data sets that have different color baselines and different photometric errors, there is one serious complication. As we have indicated, there is an unresolved, broad population of stars redward of the rMS that consists of blends, binaries, and members of the MS-a branch. Since it is unclear what relation this mixed population has with the two populations that we are studying, we wanted to exclude it from the analysis as much as possible. We did so by cutting off the reddest part of the color range, and confining our fitting to the color range that is least disturbed by the contaminated red tail.

In order to choose the red cutoff as well as possible, we gathered together all of the stars in each data set. Below we will describe for simplicity only the case of the central $3 \times 3$ mosaic of ACS images in $B$ and $R_{C}$. The procedure followed is, however, the same for the other data sets. 
Within this data set we chose the MS stars that were in the magnitude range $20.9 \leq B \leq 22.1$ (within which the two MSs are almost parallel and are maximally separated in color) and in the color range $-0.25 \leq \Delta\left(B-R_{\mathrm{C}}\right) \leq 0.25 \mathrm{mag}$. We emphasize that this ensemble of the data set, within which we will later see a considerable gradient in the relative numbers of bMS and rMS stars, will not be used to derive population results in the case of the inner ACS/WFC data set, but only to choose the red cutoff. We divided these stars into five magnitude intervals, because the observational errors, which increase the spread of the sequences, depend on magnitude. Next, we plotted histograms of the $\Delta\left(B-R_{\mathrm{C}}\right)$ distribution within each magnitude interval, using a bin size of $0.006 \mathrm{mag}$. This size is $\sim 1 / 4$ of the typical photometric error in color; it makes a good compromise between a fine enough color resolution, on the one hand, and adequate statistics, on the other hand.

The actual choice of the red cutoff is a two-tiered procedure. We must first develop a procedure for the fitting of dual Gaussians to a set of bins that has a red cutoff; then we must decide on a value of $N_{\text {red }}$, the number of bins that we include on the redder side of the red Gaussian.

Although from a mere inspection of the histograms it is clear where, approximately, the peak of the red Gaussian should lie, the narrowness of the bins leaves it uncertain in which particular bin the peak of the red Gaussian will actually fall. Since the red cutoff, $N_{\text {red }}$, is defined as being counted from that bin, we had to resort to an iterative procedure to locate the cutoff for a given value of $N_{\text {red }}$. We began by choosing a cutoff safely to the red of where we guessed that the cutoff would actually fall, and then using that cutoff in a first try at fitting the dual Gaussians. The iteration then consisted of placing the cutoff just beyond $N_{\text {red }}$ bins on the red side of the peak of the red Gaussian and fitting again; this new fit might cause the red peak to move to a different bin. When the red peak stays in the same bin, the iteration has converged; this happened after very few iterations.

We assumed trial values of $N_{\text {red }}$ from 2 to 5 , and for each of those values we iteratively computed the Gaussian parameters for each of the five magnitude intervals. We chose as the best value for $N_{\text {red }}$ the one for which the five values of $N_{\mathrm{bMS}} / N_{\mathrm{rMS}}$ were the most consistent. This value turned out to be $N_{\text {red }}=3$. With this choice made, we then moved on to fit dual Gaussians to each of our detailed data sets.

Figure 8 shows the results of this procedure. In the left panel we show our selected stars in the $B$ versus $\Delta\left(B-R_{\mathrm{C}}\right)$ diagram all of the stars this time, rather than a random selection of a fraction of them. The horizontal lines delineate our five magnitude intervals. On the right we show the final $\Delta\left(B-R_{\mathrm{C}}\right)$ histogram for each magnitude interval, and the dual-Gaussian fit to it. The individual Gaussians are shown in blue and red, respectively, and the black curve is their sum. The vertical blue and red lines are the centers of the respective Gaussians, and the vertical black line shows the red cutoff. Note that we do not show the vertical boundaries between the bins of a histogram, because on this scale they would be too close to each other. Nor do we show the Poisson errors of the counts in the bins, because they are small and would obscure the bin values themselves; the size of the errors is amply clear from the smoothness of the values in neighboring bins. The counts in the histograms are normalized so as to make the height of the red Gaussian equal to unity.

\subsection{The radial gradient of $N_{b M S} / N_{r M S}$}

Having chosen the position of the red cutoff, we were able to perform dual-Gaussian fitting on each of our data sets. Figure 9

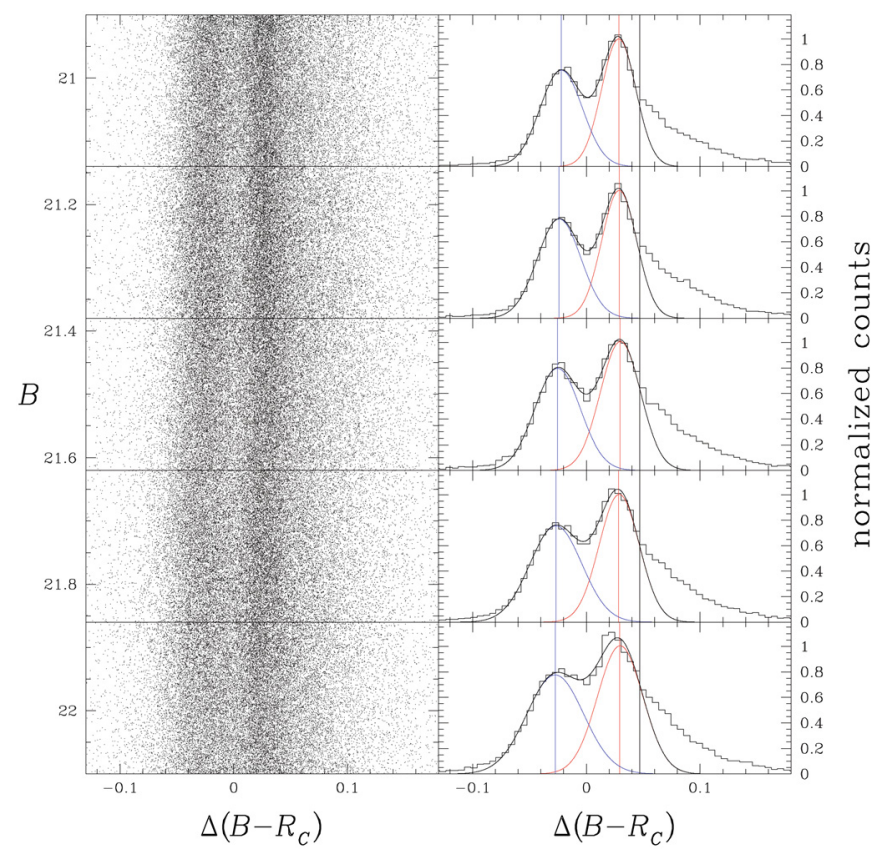

Fig. 8. (Left panel): $B$ versus $\Delta\left(B-R_{\mathrm{C}}\right)$ diagram for selected stars in our data set from the ACS/WFC $3 \times 3$ mosaic, divided into five magnitude intervals. We now plot all the stars in this sample, not just a randomly selected subset. (Right panels): $\Delta\left(B-R_{\mathrm{C}}\right)$ histograms with the Gaussian best fits. See text for details.

shows our fits. We divided the inner ACS/WFC mosaic and the outer FORS1@VLT data sets into five radial intervals for each. The intervals were chosen in such a way as to have the same number of selected stars in each of them, so that the statistical sampling errors will be uniform. (The reader should note that Fig. 9 shows all of our fields, in radial order, so that the WFPC2 field follows the inner ACS fields, and the outermost ACS field falls between two of the FORS1 fields.)

Figure 10 shows our results for the radial variation of the bMS to rMS ratio, for the five radial parts of the inner ACS mosaic, the five radial intervals of our FORS1 fields, the WFPC2 field, and the outer ACS field. Symbols of a different shape distinguish the various types of field. The outermost radial interval of the ACS/WFC mosaic is a special case, however, since it consists largely of the four corners of the mosaic, and it spans a larger radial extension. To better map the bMS/rMS distribution in this radial interval, we decided to further split it into four sub-annuli. In this way we increase the radial resolution, but pay the price of larger sampling errors. We have therefore plotted the outermost radial interval of the inner ACS/WFC mosaic twice, once as a whole annulus, and once as four sub-annuli (marked as crossed open circles in Fig. 10).

Our choice of using ellipses with fixed ellipticity and position angle to extract radial bins could have introduced some systematics in our derived $N_{\mathrm{bMS}} / N_{\mathrm{rMS}}$ ratios. To address this issue, we recalculated the $N_{\mathrm{bMS}} / N_{\mathrm{rMS}}$ ratios by extracting radial bins using simple circles, and we found no significant differences between the two radial binning methods.

Estimating the errors of our points required special attention. First we took the Poisson errors of the numbers of stars, and used them to generate Poisson errors for the values of $N_{\mathrm{bMS}} / N_{\mathrm{rMS}}$. These, however, are only a lower bound for the true error, which has additional contributions that are impossible to estimate directly; they come from blends, binaries, etc. To estimate the true errors empirically, for each value of $N_{\mathrm{bMS}} / N_{\mathrm{rMS}}$ we 


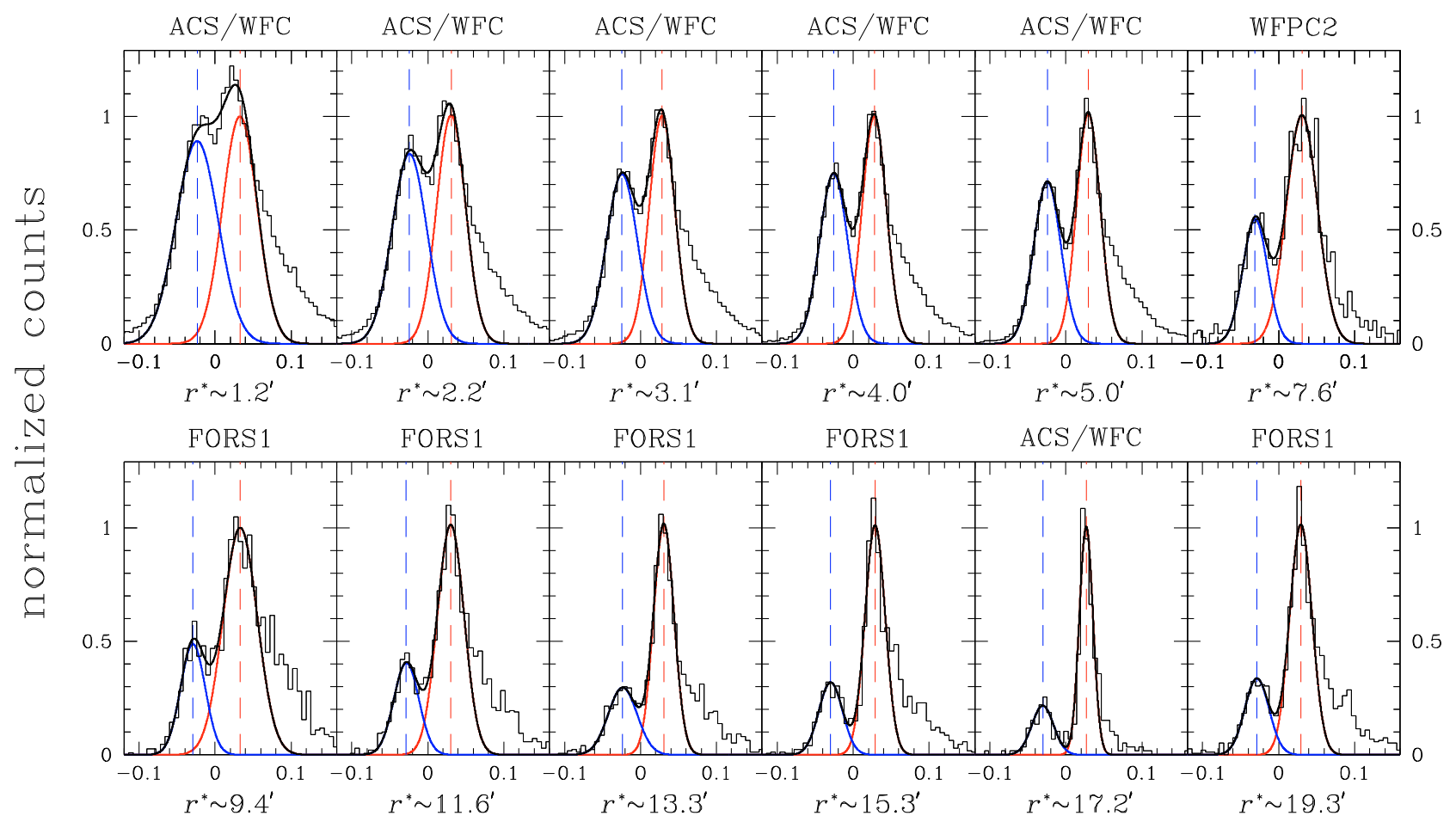

Fig. 9. Dual-Gaussians fits. As in Fig. 8, the Gaussian fits to the bMS and rMS are in blue and red respectively, and their sum in black. The vertical dashed lines mark the centers of the individual Gaussians. The individual panels are arranged in order of effective radius. (Note that all our fields are shown here, in radial order, so that the WFPC2 field follows the inner ACS fields, and the outer ACS field falls between two of the FORS1 fields.)

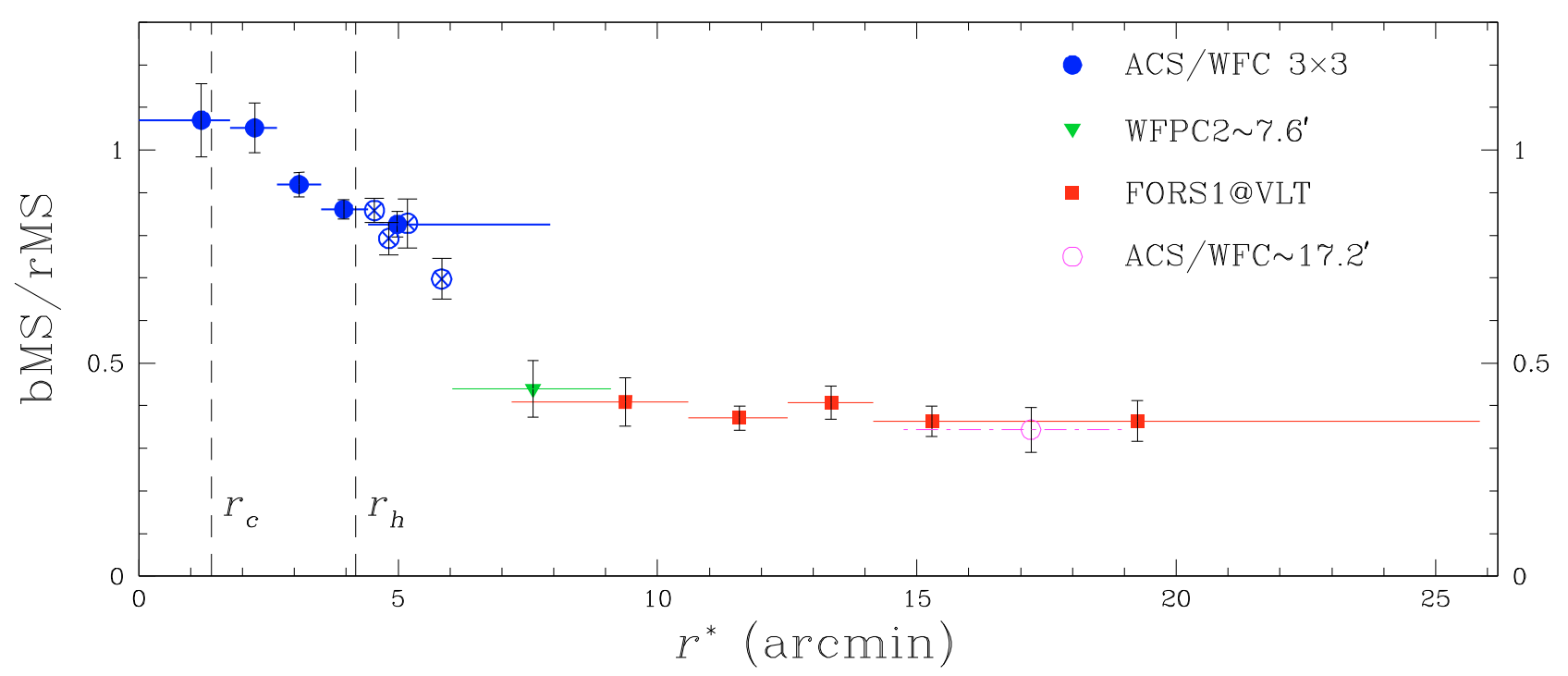

Fig. 10. $N_{\mathrm{bMS}} / N_{\mathrm{rMS}}$ ratio versus equivalent radius $r^{*}$. Different colors and symbols refer to different data sets. Dashed vertical lines mark the core radius and the half-mass radius. Error bars were calculated from the residuals of values in individual subdivisions (quadrants for the inner ACS/WFC mosaic, magnitude intervals in each outer field). To improve the radial resolution for the outermost annulus of the inner ACS/WFC mosaic, we also divided it into four sub-annuli (crossed open circles). See text for a full explanation.

subdivided the sample of stars that had been used. In the inner ACS/WFC mosaic the subsamples were the quadrants shown in Fig. 1, while for each of the outer fields, where we do not have symmetric azimuthal coverage, we divided the sample into magnitude intervals, four for each FORS1 @ VLT field and three each for the WFPC2 field and the outer ACS/WFC field.

We treated each set of subsamples as follows: Within each subsample we performed a dual-Gaussian fit, and derived from it the value of $N_{\mathrm{bMS}} / N_{\mathrm{rMS}}$. We weighted each subsample according to the number of stars in it, and took a weighted mean of the four (or three) values of $N_{\mathrm{bMS}} / N_{\mathrm{rMS}}$, to verify that this mean was equal, within acceptable round-off errors, to the value that we had found for the whole sample. (It was, within a per cent or two in nearly every case.) Finally we derived an error for the sample, from the residuals of the individual $N_{\mathrm{bMS}} / N_{\mathrm{rMS}}$ values from their mean, using the same weights as we had used for 
Table 2. Dual-Gaussian fitting results.

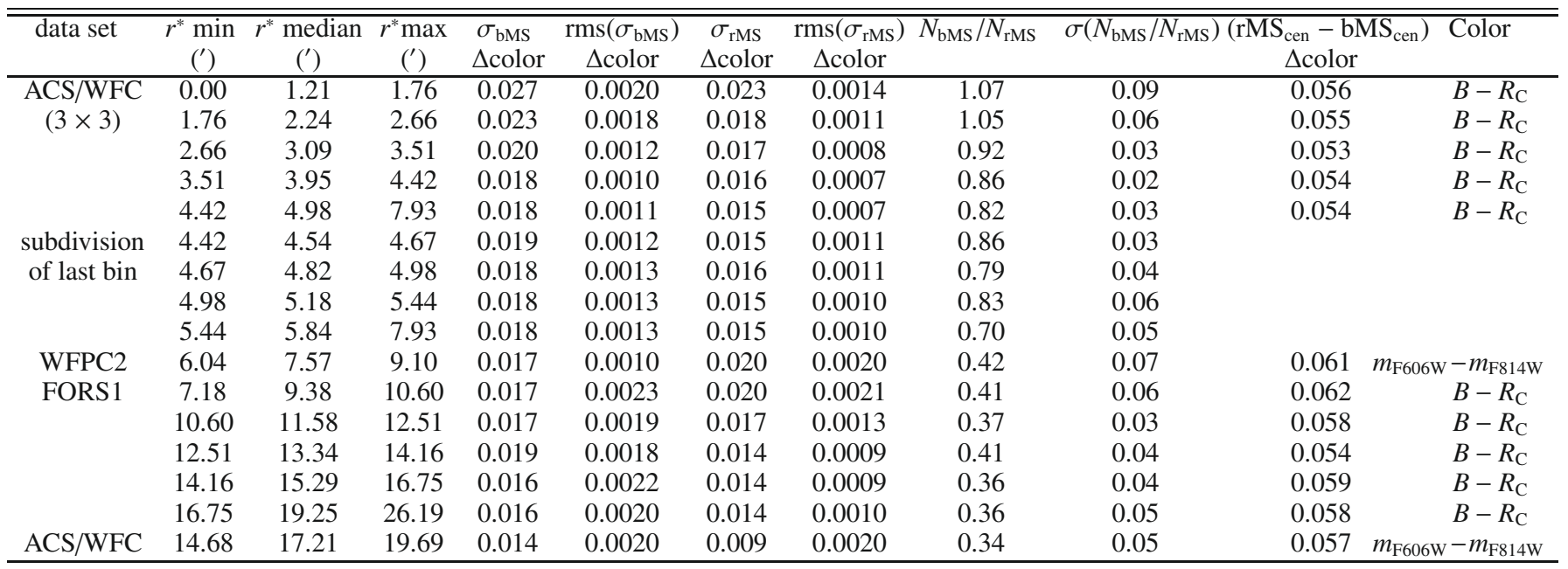

For each data set (first column) we give in Cols. 2-4 the radial extent (minimum, median, and maximum $r^{*}$ ). In Cols. 5-8 are the sigmas of the Gaussians that best fit the bMS and rMS color distributions, with errors. In the next two columns are the $N_{\mathrm{bMS}} / N_{\mathrm{rMS}}$ ratio and its error. The next-to-last column gives the color difference between the two Gaussian peaks, and the final column identifies the color baseline of the data set.

the mean. These are the error bars that are shown in Fig. 10. These errors are indeed larger than the Poisson errors, but only by about $10 \%$. We must note, however, that in addition to the random error represented by the error bars, it is likely that there is still some systematic error in our values of $N_{\mathrm{bMS}} / N_{\mathrm{rMS}}$, due to the effects of blends and binaries. On the one hand, blends have the same photometric effect as true binaries; they tend to move bMS stars into the rMS region, while many of the rMS stars that are similarly affected are eliminated by our red cut-off. This effect tends to reduce our observed value of $N_{\text {bMS }} / N_{\text {rMS }}$. It is less easy to predict, however, how such effects increase toward the cluster center. Blends, on the one hand, increase because of the greater crowding. Binaries, on the other hand, increase because their greater mass gives them a greater central concentration. To repeat, the result has been that our values of $N_{\mathrm{bMS}} / N_{\mathrm{rMS}}$ are somewhat depressed toward the cluster center, so that the gradient of $N_{\mathrm{bMS}} / N_{\mathrm{rMS}}$ that we report is probably a little lower than the real one.

Figure 10 clearly shows a strong radial trend in the ratio of bMS to rMS stars, with the bMS stars more centrally concentrated than the rMS stars. The most metal-rich population, MS-a, is too sparse, and also too hopelessly mixed with the red edge of the rMS, to allow any reliable measurement of its radial distribution, but in the next section we will examine the distribution of its progeny, RGB-a. Table 2 summarizes our results. The first column identifies the data set. Columns 2-4 give, for the inner ACS/WFC $3 \times 3$ mosaic and the FORS 1 data sets, the minimum, median, and maximum radius of the central circle or the annulus, while for the other fields these columns give the inner, median, and maximum radius that the field covers. The sigmas of the Gaussians that best fit the bMS and rMS color distributions, with their uncertainties, are in Cols. 5-8. Columns 9 and 10 give the $N_{\mathrm{bMS}} / N_{\mathrm{rMS}}$ ratio and its error. Column 11 gives the difference (in straightened color) between the peaks of the Gaussians that best fit the bMS and rMS. The last column gives the color baseline of each data set. By $\Delta$ color we mean a color difference or width, in the straightened CMD (either $\left(B, B-R_{\mathrm{C}}\right)$ or $\left(m_{\mathrm{F} 606 \mathrm{~W}}\right.$, $\left.m_{\mathrm{F} 606 \mathrm{~W}}-m_{\mathrm{F} 814 \mathrm{~W}}\right)$, whichever applies).

Our results are qualitatively consistent with those of Sollima et al. (2007), within the common region of radial coverage.
We confirm the flat radial distribution of $N_{\mathrm{bMS}} / N_{\mathrm{rMS}}$ outside $\sim 8-10$ arcmin, and a clear increase of $N_{\mathrm{bMS}} / N_{\mathrm{rMS}}$ toward the cluster center. For the first time, and as a complement to the Sollima et al. (2007) investigation, our ACS/WFC $3 \times$ 3 mosaic data set has enabled us to study the distribution of $\omega$ Cen MS stars in the innermost region of the cluster. Inside of $\sim 1.5 r_{\mathrm{c}}$ (i.e., inward of $\sim 2^{\prime}$ ), the $N_{\mathrm{bMS}} / N_{\mathrm{rMS}}$ ratio is almost flat and close to unity, with a slight overabundance of bMS stars. At larger distances from the cluster center, the $N_{\mathrm{bMS}} / N_{\mathrm{rMS}}$ ratio starts decreasing. Between $\sim 3^{\prime}$ and $\sim 8^{\prime}$ (the latter corresponding to $\sim 2$ half-mass radii) the ratio rapidly decreases to $\sim 0.4$, and remains constant in the cluster envelope. Better azimuthal and radial coverage of the region where the maximum gradient is observed would be of great value. In the radial interval between 1 and 2 half-mass radii, we can use only the corners of the ACS/WFC $3 \times 3$ mosaic, and the FORS1 photometry, which inside of $10^{\prime}$ is seriously affected by crowding and saturated stars. In any case, the star counts and even visual inspection of the histograms in Fig. 9 leave no doubt about the overall gradient.

Note that in the two innermost bins there are more bMS than rMS stars, even though the heights of the two peaks would suggest the opposite. The apparent contradiction disappears, however, when we note the much greater width of the bMS Gaussian, which more than makes up for the difference in heights. This seems to be consistent with a greater spread in chemical composition for metal-intermediate than for metal-poor stars, as first seen by Norris et al. (1997). Our approach, using a dualGaussian fit, has been optimized to estimate the value of the number ratio of bMS to rMS stars, avoiding as much as possible any contamination by blends, binaries, and MS-a stars.

We must also address the fact that the $N_{\mathrm{bMS}} / N_{\mathrm{rMS}}$ values found by Sollima et al. (2007) are consistently lower than our values. The difference is largely due to their use, on the red side, of a wide color range (see their Fig. 5) that includes nearly all of the contamination by blends, binaries, and MS-a stars that our method has so studiously avoided. This makes their numbers of rMS stars much too high - easily enough to account for their finding a value of $\sim 0.16$ in the cluster envelope, rather than our $\sim 0.4$, which is certainly much closer to the truth. Note also that we have concentrated exclusively on the ratio of numbers of 
Table 3. Results of the two artificial-star tests.

\begin{tabular}{cccccccc}
\hline \hline Field & $\left(N_{\text {bMS }} / N_{\text {rMS }}\right)_{\text {ins. }}$ & $\left(\sigma_{\text {bMS }}\right)_{\text {ins. }}$ & $\left(\sigma_{\text {rMS }}\right)_{\text {ins. }}$ & $\left(N_{\text {bMS }} / N_{\text {rMS }}\right)_{\text {rec. }}$ & $\sigma\left(N_{\text {bMS }} / N_{\text {rMS }}\right)_{\text {rec. }}$ & $\left(\sigma_{\text {bMS }}\right)_{\text {rec. }}$ & $\left(\sigma_{\text {rMS }}\right)_{\text {rec. }}$ \\
\hline central & 1.000 & 0.000 & 0.000 & 0.946 & 0.013 & 0.013 & 0.013 \\
corner & 1.000 & 0.000 & 0.000 & 0.992 & 0.013 & 0.009 & 0.009 \\
& & \multicolumn{7}{c}{ TEST2 } \\
central & 1.000 & 0.021 & 0.016 & 1.008 & 0.079 & 0.026 & 0.021 \\
corner & 1.000 & 0.016 & 0.013 & 0.996 & 0.027 & 0.019 & 0.016 \\
\hline
\end{tabular}

For each of the two fields (first column), we give in Cols. 2-4 the values of $N_{\mathrm{bMS}} / N_{\mathrm{rMS}}$ for the AS that were inserted, and the color dispersions that were given to the AS that were put on the bMS and rMS, respectively. In Cols. 5-8 are, respectively, the $N_{\mathrm{bMS}} / N_{\mathrm{rMS}}$ of the AS that were recovered, with error, followed by the sigmas of the two Gaussians that were fitted to them. See text for details.

bMS and rMS stars, making no attempt to derive absolute numbers for each component. We felt that absolute numbers would be subject to different incompleteness corrections in our different data sets, whereas the incompleteness in each data set should be the same for each component and should therefore not affect their ratio.

Finally, the robustness of our method is shown by the close agreement of our - proper-motion selected - outer ACS field (magenta open circle in Fig. 10), which has almost no crowding problems, with the outer ground-based FORS1 fields (last two red squares in Fig. 10), which are certainly affected somewhat by crowding.

\subsection{Artificial star tests}

Even with the technique that we have used to exclude the effects of photometric blends and binaries, which lie above and to the red of any MS, there is a concern that some bMS stars would be shifted into the rMS region (and some rMS stars lost on the red side of the MS), and that these shifts would distort the $N_{\mathrm{bMS}} / N_{\mathrm{rMS}}$ ratio. As a check against this possibility we have made two tests using artificial stars (AS). In each test we introduced the same AS into both the F435W and the F625W images, as follows.

For each test, we first created 45000 artificial stars, with random F435W instrumental magnitudes between -11.1 and -9.9 (corresponding to $20.9<B<21.1$ ), and random positions. We then took each of these $45000 \mathrm{AS}$, assigned it a color that placed it on the bMS, and inserted it in the F625W images, at the same position but with the $\mathrm{F} 625 \mathrm{~W}$ magnitude that corresponds to this color. We then repeated this procedure for 45000 new AS, but this time we gave them colors that put them on the rMS. (What we mean by "on" (bMS or rMS) differs between the two tests; see below for an explanation of the difference.) Each artificial star in turn was added, measured, and then removed, so as not to interfere with the other AS that were to be added after it; this procedure is that of Anderson et al. (2008), where it is explained in detail.

In order to test the effects of crowding, each of the two tests used two fields from the central $3 \times 3$ mosaic: the central field where crowding effects are maximal, and one of the corner fields, about $5 \operatorname{arcmin}\left(3.6 r_{\mathrm{c}}\right)$ southeast of the center (see Fig. 1 for a map of the $3 \times 3$ mosaic of fields).

The first of the tests (TEST1) was aimed at checking the photometric errors in the colors. To do this, we chose the color of each AS so as to put it exactly on the ridge line of the bMS or rMS; the color spread of the recovered AS would then serve as a lower-limit estimate of our photometric error.
The aim of TEST2 is to verify our ability to insert AS with $N_{\mathrm{bMS}} / N_{\mathrm{rMS}}=1$ and then recover that value, when the two MSs have intrinsic dispersions in color. To do this, we first derived the intrinsic spreads of the two sequences by taking from the fifth and seventh columns of Table 2 the simple unweighted mean of the entries in lines 1 and 2 for the central field, and in lines 4 and 5 for the corner one. (The more fastidious procedure, weighting the entry in each of the two lines according to the number of stars contributed by that annulus, would have been quite laborious and would have made no significant change in the results.) These are the observed total color spreads (intrinsic spread plus measuring error) of the bMS and rMS, respectively, in the two fields that we are using here. From these total spreads we quadratically subtracted the corresponding measuring-error spreads that we had found in TEST1, so as to get estimates of the intrinsic color spreads of the two sequences. We created AS in the same manner as in TEST1, but this time instead of placing the AS on the center lines of bMS and rMS, we adjusted the F625W magnitudes so as to give the AS a Gaussian spread in color around each sequence, using the intrinsic sigmas that we had just found. After the measuring process, these AS should duplicate the observed total spreads, and can be used to estimate the amount of contamination between the two main sequences. To repeat, each test was performed both on both the central and the corner field.

The results of these AS tests are summarized in brief numerical form in Table 3 and in graphical form in Figs. 11 and 12. In each figure the left and right halves refer to the central and corner fields, respectively, while each half figure is divided into three panels that show, from left to right, the CMD, the straightened $\mathrm{CMD}$, and the decomposition of the number densities of the latter into best-fitting Gaussians. Each panel showing the Gaussian fits is subdivided into five magnitude intervals (very similarly to what is done in Fig. 8). Columns 2-4 of Table 3 give, for each field and AS test, the $N_{\mathrm{bMS}} / N_{\mathrm{rMS}}$ ratio of the inserted AS and the dispersions of the MSs. The recovered values (weighted mean of the five magnitude bins and its error, as explained in detail for real stars in Sect. 3.3) are shown in Cols. 5-8.

From the results of TEST1 we conclude that in each field the color spread introduced by measuring error is the same for bMS stars as for the rMS, and that it is about $40 \%$ higher in the central field than in the less-crowded corner field. TEST1 has served two purposes: (1) it gave us a clear, effective measure of the effect of crowding on the color spread. (2) It evaluated the color spreads due to measuring error alone, which we used in setting up TEST2. (Its results for $N_{\mathrm{bMS}} / N_{\mathrm{rMS}}$ are given, pro forma, but they have no real significance, since the color spreads used in TEST1 are so narrow that our color bin-width does not sample them adequately.) It is TEST2 which directly tests our previous conclusions about the size of $N_{\mathrm{bMS}} / N_{\mathrm{rMS}}$. We conclude 

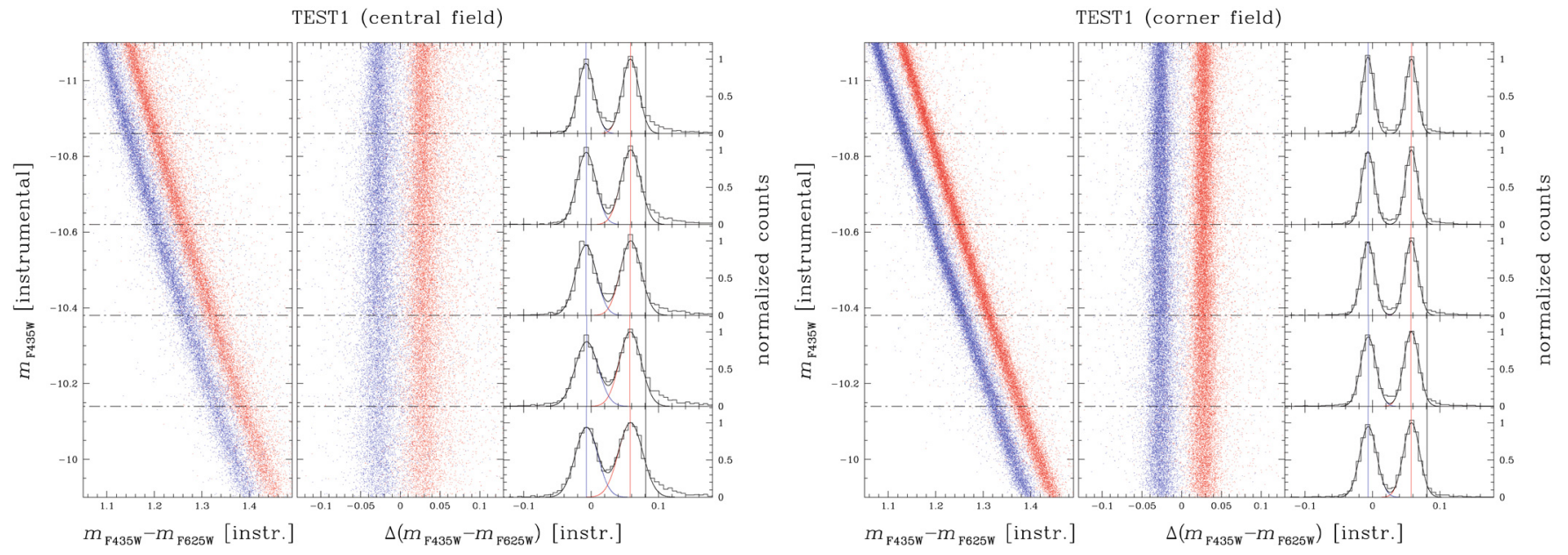

Fig. 11. TEST1 artificial star analysis for the central ACS/WFC $3 \times 3$ mosaic field (left panels), and for the corner field (right panels). For each panel, we show the CMD with the recovered stars (in blue for the bMS stars and in red for the rMS stars), for five magnitude intervals. The straightened MSs are plotted in the middle, while on the right we show the color histograms, with the dual-Gaussian fits. The vertical lines in blue, red, and black mark, respectively, the centers of the two Gaussians and the red cut-off. See text for details.

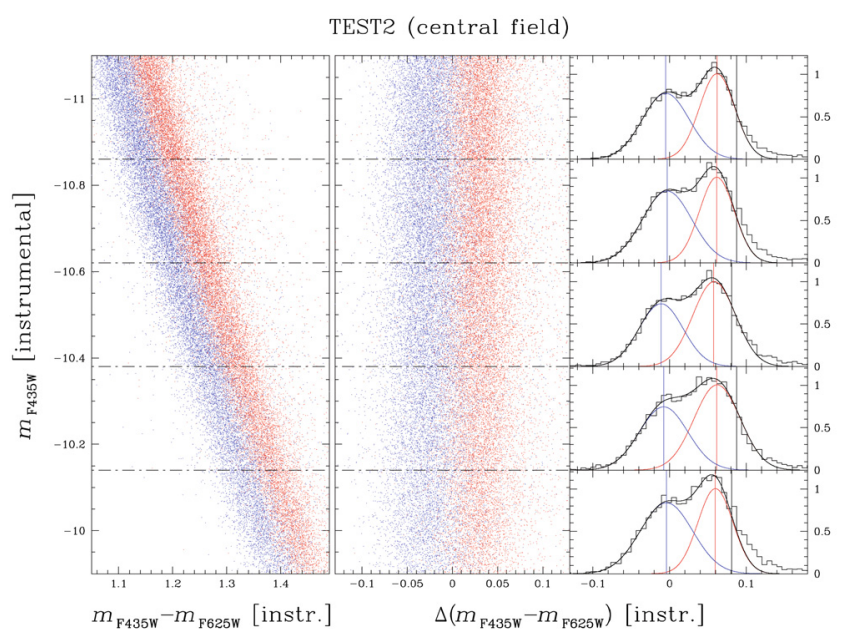

Fig. 12. Same as Fig. 11, but now for TEST2. See text for details.

from it that the AS tests recover our input values of $N_{\mathrm{bMS}} / N_{\mathrm{rMS}}$, within the uncertainties of the measurement.

In this section we have demonstrated, on two extreme fields of the ACS inner mosaic, that our dual-Gaussian fitting method is fully effective in overcoming the effects of crowding on the distribution of colors, and that it reliably estimates the relative star numbers in the two sequences. (Note that we use this same method for all of our other data sets too.) As we noted at the end of Sect. 3.3, the excellent agreement between the results from our completely uncrowded outer ACS field and those from our outer FORS1 fields establishes the validity of the latter, without recourse to any additional AS texts for them.

\section{Radial gradients in the RGB subpopulations}

It has been known since the end of the sixties that the RGB of $\omega$ Cen is broader than would be expected from photometric errors (Woolley \& Dickens 1967), but it was only in 1999 that Lee et al. (1999) clearly detected at least two distinct RGBs. Later on, Pancino et al. (2000) demonstrated that there is a correlation between the photometric peaks across the RGB and three peaks in the metallicity distribution. On this basis, they defined the three RGB groups: RGB-MP, RGB-MInt, and RGB-a, characterized

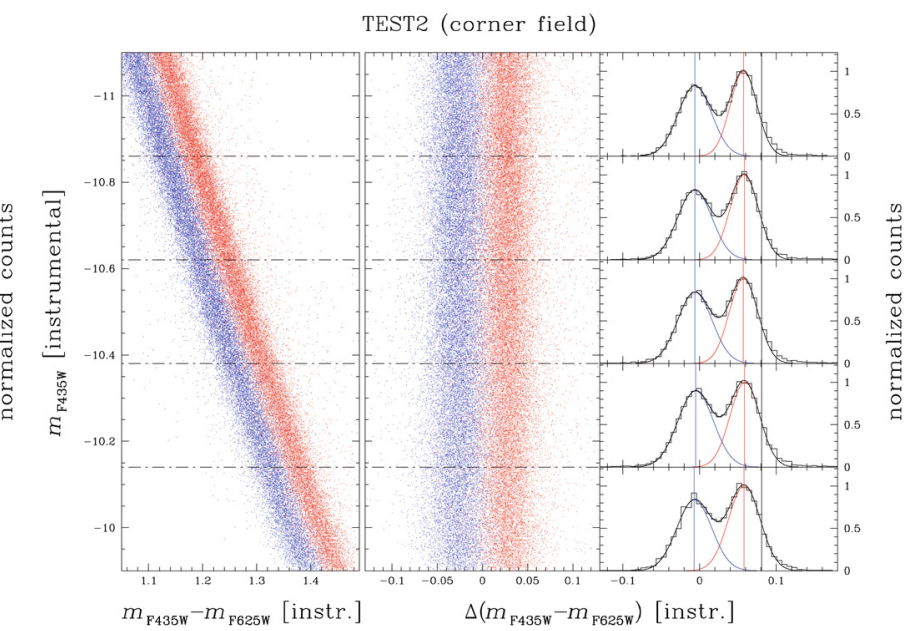

by an increasing metallicity. In this section we will present a detailed study of the radial distributions of these components.

\subsection{Defining the RGB-MP, RGB-MInt, and RGB-a subsamples}

Unfortunately the WFPC2, FORS1, and outer ACS/WFC data sets we used to analyze the main-sequence population in the previous section are saturated even at the MS turn-off level, and are therefore unusable for the study of the RGB radial distributions.

Our WFI@2.2m photometric and proper-motion catalog (Bellini et al. 2009), however, is an excellent data base for this study, particularly in view of the fact that we can safely remove field objects in the foreground and background, thanks to our accurate proper motions. This proper-motion cleaning is of fundamental importance in the outer envelope of the cluster, where there can be more field stars than cluster giants. In the central regions of the cluster, the WFI@2.2m data are less accurate due to the poorer photometry caused by the crowded conditions, so there we take advantage of our high-resolution inner ACS/WFC $3 \times 3$ mosaic, which included short exposures 
to measure the bright stars. Below we describe how we extracted the $\omega$ Cen RGB subsamples from these two data sets.

Because of the complex distribution of the stars along the RGB we were forced to use bounding boxes to select the different RGBs. This selection procedure is less accurate than what we were able to do for the bMS and the rMS; nevertheless it is still accurate enough to study the general trend of the radial distribution of the relative numbers of RGB-MP, RGB-Mint, and RGB-a stars. The Poisson error from the smaller number of RGB stars makes the more precise procedure less critical.

For the ACS data, we defined bounding boxes for the RGB subpopulations of $\omega$ Cen in the CMD obtained from the data set of the ACS/WFC $3 \times 3$ mosaic, for which the largenumber statistics make the separation among the different RGBs easier to see. We extracted three RGB subpopulations, in a way very similar to that used by Ferraro et al. (2002). (Note that other authors, e.g. Rey et al. 2004; Sollima et al. 2005a; Johnson et al. 2009, have defined four or even five RGB subpopulations.) The left panel of Fig. 13 shows the three RGB bounding-box regions drawn in the CMD from the ACS/WFC $3 \times 3$ mosaic, to identify the three subgroups RGB-MP, RGB-MInt, and RGBa. Our RGB selections are limited to magnitudes brighter than $B=17.9$, and contain 5184 RGB-MP stars, 4379 RGB-MInt stars, and 383 RGB-a stars.

In extracting the RGB subpopulations from our WFI@2.2m data set we chose to define the subpopulations in the $B, B-V$ CMD. Even though we cannot adopt exactly the same selection boxes in the $B, B-R_{\mathrm{C}} \mathrm{CMD}$ as for the ACS/WFC $3 \times 3$ mosaic. This choice might appear awkward, not only because the color baseline $B-V$ is shorter than the $B-R_{\mathrm{C}}$ baseline, but also because the WFI $R_{\mathrm{C}}$ filter is very similar to the ACS/WFC F625W filter. There are other good reasons for adopting the $B-V$ color baseline, however. The most important one is that the WFI photometry obtained with the $V$ filter has ten times as much integration time, and more dithered images than those available for the $R_{\mathrm{C}}$ filter. Therefore our $V$ photometry is considerably more precise, and more accurate, than our $R_{\mathrm{C}}$ magnitudes. Moreover, our empirical sky-concentration correction (very important for such studies) is better defined in $V$ than in $R_{\mathrm{C}}$ (see Bellini et al. 2009).

In this WFI@2.2m $B$ vs. $B-V \mathrm{CMD}$, we tried to define the bounding boxes in a way that was as consistent as possible with what we did for the data set from ACS/WFC $3 \times 3$ mosaic. We cross-identified the stars that are in common between the sample that we had selected from the RGB CMD of the ACS/WFC $3 \times 3$ mosaic, on the one hand, and the WFI@2.2m $B-V$ data set on the other hand, and we carefully drew by hand, in the $(B, B-V) \mathrm{CMD}$, bounding boxes that would include the same stars as in the sample from the ACS/WFC $3 \times 3$ mosaic.

In addition, we selected from the WFI@2.2m data set the stars that were measured best (both photometrically and astrometrically), and were most likely to be members of $\omega$ Cen. To make the selection we used the error quantities in Cols. 7 , 9,13 , and 15 of Table 6 of Bellini et al. (2009). These are the errors of the two components of proper motion and of the $B$ and $V$ magnitudes. Our selection consisted of choosing, at the bright end of the RGB, stars whose proper-motion error has a magni-

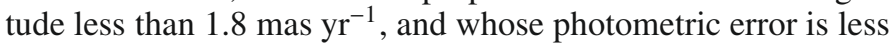
than 0.02 mag in each band; we also required that the proper motion of a star differs from the mean motion of cluster stars by no more than 2.1 mas $\mathrm{yr}^{-1}$. At the faint end of the RGB we allowed

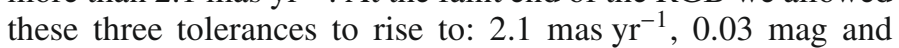
$3.8 \mathrm{mas} \mathrm{yr}^{-1}$, respectively. This high-quality data set comprised 4993 RGB-MP stars, 3057 RGB-MInt, and 292 RGB-a stars.

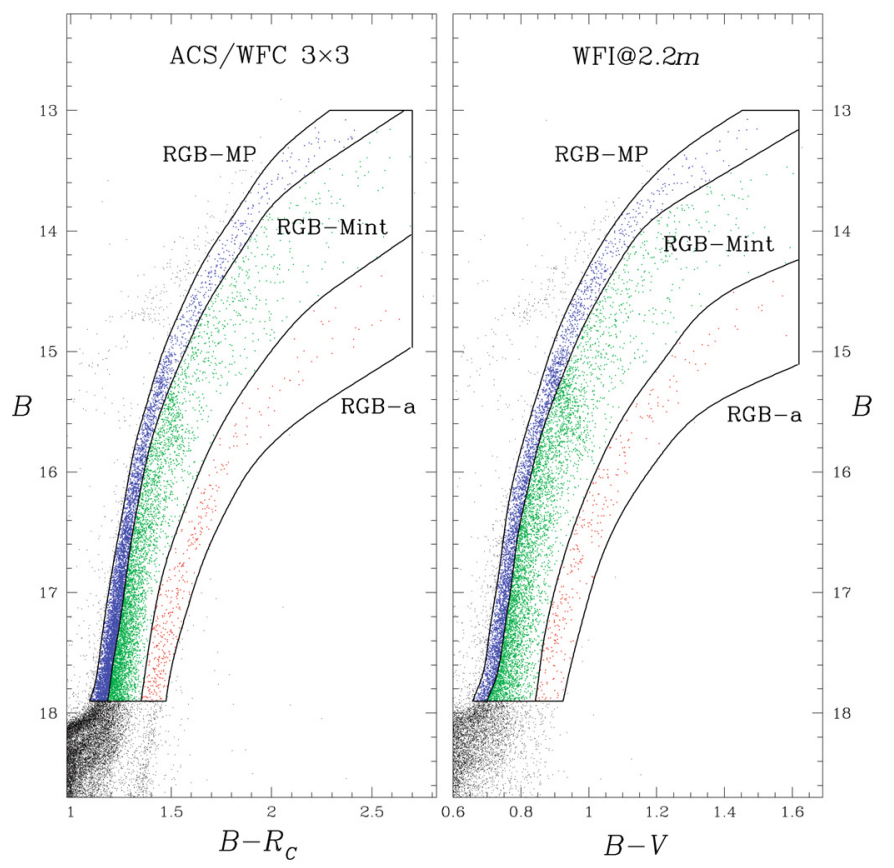

Fig. 13. CMDs of the $\omega$ Cen RGB from ACS/WFC $3 \times 3$ mosaic ( $B$ vs. $B-R_{\mathrm{C}}$, left panel) and from WFI@2.2m data ( $B$ vs. $B-V$, right panel). The RGB subpopulations selected are also plotted with different colors. See text for details.

The right-hand panel of Fig. 13 shows the WFI@2.2m RGB subpopulations that were selected in this way. We note that whereas the RGB-a sample is well separated from the other two RGB components, the RGB-MP and RGB-MInt components are separated only by an arbitrary dividing line, so that small differences in defining the bounding boxes might result in some cross-contamination in those two samples.

\subsection{Relative radial distributions of RGB stars}

We divided our WFI@2.2m data set into ten radial bins, each containing approximately the same number of RGB-MInt stars, and the ACS/WFC $3 \times 3$ data set into five radial bins, again with the same equal-number criterion. For each of these bins we counted the number of RGB stars in each subpopulation.

In Fig. 14 we show the derived radial gradients. As it has not been possible to perform the same error analysis as was done for the MS stars (because of the much smaller number of stars), the error bars in Fig. 14 represent only Poisson errors, and should be considered a lower limit to the real errors. In panel (a) we show the radial distribution of the ratio RGBa/RGB-MInt. Blue full circles refer to the ACS/WFC $3 \times 3$ data set, and red triangles to the WFI@2.2m data. Vertical dashed lines mark the core radius $r_{\mathrm{c}}$ and the half-mass radius $r_{\mathrm{h}}$. We found that, within the errors, the RGB-a and the RGB-MInt stars share the same radial distribution, since their ratio is constant over the entire radial range covered by our two data sets. In panel (b), we plot the ratio RGB-MInt/RGB-MP for the two data sets. The RGB-MInt stars are more centrally concentrated than the RGB-MP stars, with a flatter trend within $\sim 1 r_{\mathrm{h}}$, a rapid decline out to $\sim 8^{\prime}-10^{\prime}$, and again a flat relative distribution outside. There is a hint, also, that the RGB-MInt/RGB-MP ratio could be nearly constant within the half-mass radius. We find that the general radial trend of the RGB-MInt/RGB-MP star-count ratio is 


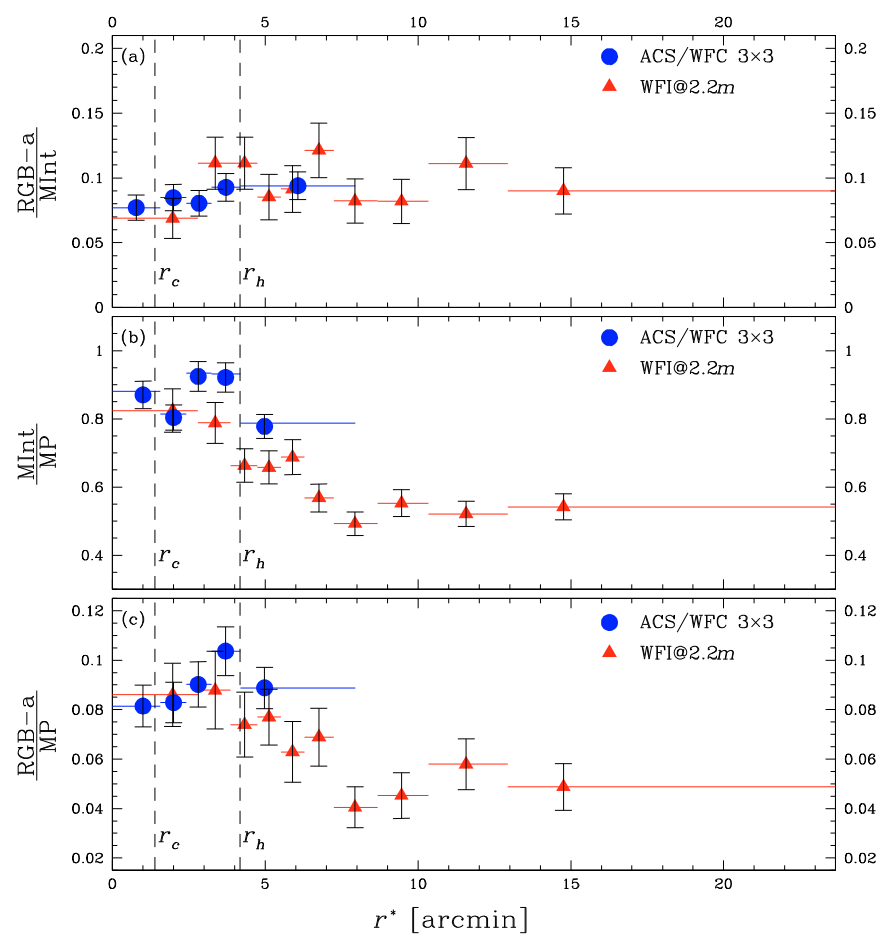

Fig. 14. (a) Radial distribution of the ratio RGB-a/RGB-MInt for the WFI@2.2m data set (red triangles) and for the ACS/WFC $3 \times$ 3 data set (blue circles). Vertical dashed lines mark the core radius and the half-mass radius, respectively. (b) Radial distribution of the ratio RGB-MInt/RGB-MP. (c) Radial distribution of the ratio RGB-a/RGB-MP. See text for details.

consistent with that of $N_{\mathrm{bMS}} / N_{\mathrm{rMS}}$. This result provides additional evidence (in agreement with the metallicity measurements by Piotto et al. 2005) that the bMS and the RGB-MInt population must be part of the same group of stars, with the same metal content and the same radial distribution within the cluster. Panel (c) shows that the ratio RGB-a/RGB-MP resembles, within the errors, the RGB-MInt/RGB-MP trend. We were unable to examine this trend for the MS part of the RGB-a population, since the MS-a sequence cannot be followed below $B \sim 20$.

Our analysis confirms the results by Norris et al. (1997), Hilker \& Richtler (2000), Pancino et al. (2000), Rey et al. (2004), and Johnson et al. (2009), who found that the most metalpoor RGB stars are less concentrated than the RGB-MInt ones. Moreover, we can also confirm that the RGB-a and the RGBMInt share the same radial distribution within $\omega \mathrm{Cen}$, as found by Norris et al. (1997), Pancino et al. (2000), and Pancino et al. (2003) for RGB-a only.

It is important to note that because we were able to use proper motions to construct a pure cluster sample, our results are not affected by field-star contamination, which would tend to enhance the RGB-a star counts in the cluster outskirts with respect to the more populous RGB-MP sample (which also covers a smaller region in the CMD). Field-star contamination is likely the reason that Hilker \& Richtler (2000) and Castellani et al. (2007) found the RGB-a/RGB-MP ratio to increase with distance from the cluster center - the opposite trend from what is seen here. Moreover, it is interesting to note that the different RGB-Mint subgroups (as highlighted, e.g., by Sollima et al. 2005a) might well have a different radial behavior, but necessarily - since we cannot distinguish them in the CMD - we have to treat them together and study only their average gradient.

\section{Discussion}

In this paper we have analyzed the radial distribution of the different MS and RGB components in the globular cluster $\omega$ Centauri. We used high-resolution ACS/WFC images to study the inner regions of the cluster, and ACS/WFC, WFPC2 and FORS1@VLT images, as well as WFI@2.2m images, for the cluster envelope. We found that there are slightly more bMS stars than rMS stars in the inner 2 core radii. At larger distances from the cluster center, out to $\sim 8$ arcmin, the relative number of $N_{\mathrm{bMS}} / N_{\mathrm{rMS}}$ stars drops sharply, and then remains constant at $N_{\mathrm{bMS}} / N_{\mathrm{rMS}} \sim 0.4$, out to half the tidal radius of the cluster.

Our most precise photometry comes from the outer ACS field at $17^{\prime}\left(12 r_{\mathrm{c}}\right)$, where we find that the color dispersion $(\sigma)$ of the bMS is about $50 \%$ larger than that of the rMS. The other observations are consistent with this, though they are unable to measure $\sigma$ so precisely, on account of crowding (in the inner ACS field) and other errors (in the ground-based fields).

The RGB-MInt population (associated with the bMS by Piotto et al. 2005) and the RGB-MP sample (which includes the progeny of the rMS) follow a trend similar to that of $N_{\mathrm{bMS}} / N_{\mathrm{rMS}}$. The most metal-rich component of the RGB, RGB-a, also follows the same distribution as the RGB-MInt component.

On the hypothesis that the bMS, the presumably helium-rich population, is a second generation of stars formed by the lowvelocity material ejected by a primordial population (which we assume to be the more metal-poor rMS population), the bMS must have formed from matter that collected in the cluster center via some kind of cooling flow. This is in qualitative agreement with the recent models by Bekki \& Norris (2006) and D'Ercole et al. (2008). The very long relaxation time (halfmass relaxation time longer than $10 \mathrm{Gyr}$, according to the Harris 1996 compilation) has preserved some information about the original kinematic and spatial distribution of the material from which the younger component took form. Interestingly enough, the third, most-metal-rich population is also more concentrated than the most metal-poor component, and has a radial distribution that is rather similar to that of the intermediate-metallicity sample. It is also noteworthy that the bMS component has a broader color distribution than the rMS one. This fact may reflect, at least in part, the large dispersion in iron abundance of the intermediate-metallicity component (e.g. Norris \& Da Costa 1995). Alternatively, this bMS spread could be an indication of the dispersion of other chemical elements, including He. Only a detailed analysis of the metal content of the two MSs can solve this issue, but for this we might need to wait for the next generation of $30+m$ telescopes, on account of the faintness of these stars.

Acknowledgements. A.B. acknowledges support by the CA.RI.PA.RO. foundation, and by STScI under the 2008 graduate research assistantship program. I.R.K. and J.A. acknowledge support by STScI under grants GO-9442, GO-9444, and GO-10101. G.P. and A.P.M. acknowledge partial support by MIUR under the program PRIN2007 (prot. 20075TP5K9) and by ASI under the program ASI-INAF I/016/07/0.

\section{References}

Anderson, J. 1997, Ph.D. Thesis, Univ. of California, Berkeley

Anderson, J. 2002, in Omega Centauri, A Unique Window into Astrophysics, ed. F. van Leeuwen, J. D. Hughes, \& G. Piotto (San Francisco: ASP), ASP Conf. Ser., 265, 87

Anderson, J., \& King, I. R. 2000, PASP, 112, 1360

Anderson, J., \& King, I. R. 2006, ACS/ISR 2006-01 (Baltimore: STSci)

Anderson, J., \& van der Marel, R. P. 2009, ApJ, submitted [arXiv: 0905. 0627]

Anderson, J., Bedin, L. R., Piotto, G., Yadav, R. S., \& Bellini, A. 2006, A\&A, 454, 1029 
Anderson, J., Sarajedini, A., Bedin, L. R, et al. 2008, AJ, 135, 2055

Anderson, J., Piotto, G., King, I. R., Bedin, L. R., \& Guhathakurta, P. 2009, ApJ, 697, L58

Bedin, L. R., Piotto, G., Anderson, J., et al. 2004, ApJ, 605, L125

Bedin, L. R., Cassisi, S., Castelli, F., et al. 2005, MNRAS, 357, 1038

Bellazzini, M., Ibata, R. A., Chapman, S. C., et al. 2008, AJ, 136, 1147

Bellini, A., Piotto, G., Bedin, L. R., et al. 2009, A\&A, 493, 959

Bekki, K., \& Norris, J. E. 2006, ApJ, 637, L109

Bekki, K., \& Mackey, A. D. 2009, MNRAS, 394, 124

Bessell, M. S., \& Norris, J. 1976, ApJ, 208, 369

Butler, D., Dickens, R. J., \& Epps, E. 1978, ApJ, 225, 148

Calamida, A., Stetson, P. B., Bono, G., et al. 2005, ApJ, 634, L69

Cannon, R. D., \& Stobie, R. S. 1973, MNRAS, 162, 207

Carretta, E., Bragaglia, A., Gratton, R. G., et al. 2006, A\&A, 450, 523

Carretta, E., Bragaglia, A., Gratton, R. G., \& Lucatello, S. 2008 [arXiv: 0811.3591v1]

Castellani, V., Calamida, A., Bono, G., et al. 2007, ApJ, 663, 1021

D'Antona, F., \& Caloi, V. 2004, ApJ, 611, 871

D’Antona, F., Bellazzini, M., Caloi, V., et al. 2005, ApJ, 631, 868

D'Ercole, A., Vesperini, E., D’Antona, F., McMillan, S. L. W., \& Recchi, S. 2008, MNRAS, 391, 825

Decressin, T., Baumgardt, H., \& Kroupa, P. 2008, A\&A, 492, 101

Dinescu, D. I., Girard, T. M., \& van Altena, W. F. 1999, AJ, 117, 1792

Faria, D., Johnson, R. A., Ferguson, A. M. N., et al. 2007, AJ, 133, 1275

Ferraro, F. R., Bellazzini, M., \& Pancino, E. 2002, ApJ, 573, L95

Ferraro, F. R., Sollima, A., Pancino, E., et al. 2004, ApJ, 603, L81

Freeman, K. C., \& Rodgers, A. W. 1975, ApJ, 201, L71

Freeman, K. C. 1993, in The Globular Cluster-Galaxy Connection, ed. G. H. Smith, \& J. P. Brodie (San Francisco: ASP), ASP Conf. Ser., 48, 608

Freyhammer, L. M., Monelli, M., Bono, G., et al. 2005, ApJ, 623, 860

Geyer, E. H., Nelles, B., \& Hopp, U. 1983, A\&A, 125, 359

Gilliland, R. 2004, ACS/ISR 2004-01 (Baltimore: STScI)

Harris, W. E. 1996, AJ, 112, 1487, as updated in February, 2003

Hilker, M., \& Richtler, T. 2000, A\&A, 362, 895

Hilker, M., Kayser, A., Richtler, T., \& Willemsen, P. 2004, A\&A, 422, L9

Holtzman, J. A., Burrows, C. J., Casertano, S., et al. 1995, PASP, 107, 1065

Ideta, M., \& Makino, J. 2004, ApJ, 616, L107

Johnson, C. I., Pilachowski, C. A., Rich, R. M., \& Fulbright, C. P. 2009, ApJ, 698, 2048

Lee, Y.-W., Joo, J.-M., Sohn, Y.-J., et al. 1999, Nature, 402, 55

Makino, J., Akiyama, K., \& Sugimoto, D. 1991, Ap\&SS, 185, 63

Manfroid, J., \& Selman, F. 2001, The Messenger, 104, 16

Marino, A. F., Villanova, S., Piotto, G., et al. 2008, A\&A, 490, 625

Marino, A. F., Milone, A. P., Piotto, G., et al. 2009, A\&A, 505, 1099

Milone, A. P., Bedin, L. R., Piotto, G., et al. 2008, ApJ, 673, 241

Milone, A. P., Bedin, L. R., Piotto, G., \& Anderson, J. 2009, A\&A, 497, 755

Moretti, A., Piotto, G., Arcidiacono, C., et al. 2009, A\&A, 493, 539

Norris, J. E. 2004, ApJ, 612, L25
Norris, J., \& Bessell, M. S. 1975, ApJ, 201, L75

Norris, J., \& Bessell, M. S. 1977, ApJ, 211, L91

Norris, J. E., \& Da Costa, G. S. 1995, ApJ, 441, L81

Norris, J. E., Freeman, K. C., \& Mighell, K. J. 1996, ApJ, 462, 241

Norris, J. E., Freeman, K. C., Mayor, M., \& Seitzer, P. 1997, ApJ, 487, L187

Pancino, E., Ferraro, F. R., Bellazzini, M., Piotto, G., \& Zoccali, M. 2000, ApJ, 534, L83

Pancino, E., Seleznev, A., Ferraro, F. R., Bellazzini, M., \& Piotto, G. 2003, MNRAS, 345, 683

Pancino, E., Galfo, A., Ferraro, F. R., \& Bellazzini, M. 2007, ApJ, 661, L155

Piotto, G. 2009, in The Ages of Stars, ed. E. E. Mamajek, D. R. Soderblom, \&

R. F. G. Wyse (Cambridge: Cambridge University Press), IAU Symp., 258, 233

Piotto, G., Villanova, S., Bedin, L. R., et al. 2005, ApJ, 621, 777

Piotto, G., Bedin, L. R., Anderson, J., et al. 2007, ApJ, 661, L53

Platais, I., Wyse, R. F. G., Hebb, L., Lee, Y.-W., \& Rey, S.-C. 2003, ApJ, 591, L127

Renzini, A. 2008, MNRAS, 391, 354

Renzini, A., \& Fusi Pecci, F. 1988, ARA\&A, 26, 199

Rey, S.-C., Lee, Y.-W., Ree, C. H., et al. 2004, AJ, 127, 958

Rich, R. M., Reitzel, D. B., Guhathakurta, P., Gebhardt, K., \& Ho, L. C. 2004, AJ, 127, 2139

Sarajedini, A., \& Layden, A. C. 1995, AJ, 109, 1086

Sarajedini, A., Bedin, L. R., Chaboyer, B., et al. 2007, AJ, 133, 1658

Searle, L. 1977, in Evolution of Galaxies and Stellar Populations, ed. B. M. Tinsley, \& R. B. Larson (New Haven: Yale Univ. Obs.), 219

Siegel, M. H., Dotter, A., Majewski, S. R., et al. 2007, ApJ, 667, L57

Sirianni, M., Jee, M. J., Benítez, N., et al. 2005, PASP, 117, 1049

Stetson, P. B. 1987, PASP, 99, 191

Stetson, P. B. 1994, PASP, 106, 250

Stetson, P. B. 2000, PASP, 112, 925

Stetson, P. B. 2005, PASP, 117, 563

Sollima, A., Ferraro, F. R., Pancino, E., \& Bellazzini, M. 2005a, MNRAS, 357, 265

Sollima, A., Pancino, E., Ferraro, F. R., et al. 2005b, ApJ, 634, 332

Sollima, A., Ferraro, F. R., Bellazzini, M., et al. 2007, ApJ, 654, 915

Stanford, L. M., Da Costa, G. S., Norris, J. E., \& Cannon, R. D. 2006, ApJ, 653, L117

Suntzeff, N. B., \& Kraft, R. P. 1996, AJ, 111, 1913

Tsuchiya, T., Korchagin, V. I., \& Dinescu, D. I. 2004, MNRAS, 350, 1141

van de Ven, G., van den Bosch, R. C. E., Verolme, E. K., \& de Zeeuw, P. T. 2006, A\&A, 445, 513

van der Marel, R. P., \& Anderson, J. 2009, ApJ, submitted [arXiv: 0905. 0638]

Villanova, S., Piotto, G., King, I. R., et al. 2007, ApJ, 663, 296

Woolley, R. V. d. R., \& Dickens, R. J. 1967, Roy. Obs. Bull., 128

Zinnecker, H., Keable, C. J., Dunlop, J. S., Cannon, R. D., \& Griffiths, W. K. 1988, in The Harlow-Shapley Symposium on Globular Cluster Systems in Galaxies, ed. J. E. Grindlay, \& A. G. D. Philip (Cambridge: Cambridge University Press), IAU Symp., 126, 603 